\title{
1 Persistence of quantal synaptic vesicle recycling following dynamin depletion
}

2

3 Olusoji A.T. Afuwape ${ }^{1}$, Natali L. Chanaday ${ }^{2}$, Merve Kasap ${ }^{2}$, Lisa M. Monteggia ${ }^{2,3}$ and Ege T.

4 Kavalali ${ }^{2,3, *}$

$5{ }^{1}$ Department of Neurosurgery, University of Arkansas for Medical Sciences, 4301 W. Markham

6 street, Little Rock, AR 72205

$7 \quad{ }^{2}$ Department of Pharmacology, Vanderbilt University, Nashville, TN 37240-7933, USA

$8 \quad{ }^{3}$ Vanderbilt Brain Institute, Vanderbilt University, Nashville, TN 37240-7933, USA

9

10 * Corresponding author: (ege.kavalali@vanderbilt.edu)

11 Ege T. Kavalali, Department of Pharmacology, Vanderbilt University, Nashville, TN 37240.

12 Phone: 615-343-5480. E-mail: ege.kavalali@vanderbilt.edu 


\section{Abstract}

15 Dynamins are GTPases required for pinching vesicles off the plasma membrane once a critical

16 curvature is reached during endocytosis. Here, we probed dynamin function in central synapses

17 by depleting all three dynamin isoforms in postnatal hippocampal neurons. We found a

18 decrease in the propensity of evoked neurotransmission as well as a reduction in synaptic

19 vesicle numbers. Using the fluorescent reporter vGluT1-pHluorin, we observed that

20 compensatory endocytosis after $20 \mathrm{~Hz}$ stimulation was arrested in $\sim 40 \%$ of presynaptic

21 boutons, while remaining synapses showed only a modest effect suggesting the existence of a

22 dynamin-independent endocytic pathway in central synapses. Surprisingly, we found that the

23 retrieval of single synaptic vesicles, after either evoked or spontaneous fusion, was largely

24 impervious to disruption of dynamins. Overall, our results suggest that classical dynamin-

25 dependent endocytosis is not essential for retrieval of synaptic vesicle proteins after quantal

26 single synaptic vesicle fusion. 


\section{Introduction}

The robustness of synaptic transmission relies on the ability of retrieving, reforming and refilling synaptic vesicles after their fusion in a reliable and swift manner (Chanaday and Kavalali, 2017). Although the molecular machinery responsible for regulated secretion and endocytosis is conserved among species and cell types, some components have evolved to be more specific for neurons (Saheki and De Camilli, 2012; Soykan et al., 2016). During endocytosis, a critical step involves scission of the nascent vesicle from the plasma membrane. The observation of depleted synaptic vesicles and arrested budding endosomes in the shibire mutant fly (Koenig and Ikeda, 1989) led to the discovery of the GTPase dynamin as the essential protein catalyzing the fission of membranes (Antonny et al., 2016; Ferguson and De Camilli, 2012).

While Drosophila has a single gene for dynamin, mammalians have three dynamin isoforms, all of which are expressed in the nervous system and are present at the synapse (Ferguson and De Camilli, 2012). Defects in synaptic vesicle recycling during high frequency stimulation have been described after deletion of the most abundant dynamin isoforms - dynamin 1 and 3 - in the nervous system; however, synaptic vesicle endocytosis persisted partly due to the more ubiquitously expressed isoform dynamin 2 (Ferguson et al., 2007; Raimondi et al., 2011). In superior cervical ganglion neurons, knock down of dynamin 2 revealed defects in rapid recovery of the readily releasable pool of synaptic vesicles after high frequency stimulation (Tanifuji et al., 2013). A similar finding was reported in chromaffin cells where after prolonged stimulation, a dynamin 2 dependent endocytosis mechanism was activated to retrieve vesicles (Artalejo et al., 2002). A function for dynamin 2 at the synapse is also supported by the finding that $\mathrm{Ca}^{2+}$ influx in neurons inhibits dynamin mediated endocytosis at the active zone and has been shown to reduce specifically dynamin 2 GTPase activity in HeLa cells (Cousin and Robinson, 2000). Taken together, all the different isoforms of dynamin have been functionally implicated in synaptic vesicle endocytosis with varying degrees of importance but with substantial overlap among them.

Assessing the role of all dynamin isoforms has been challenging due to the non-viability of dynamin triple knock out (TKO) animals. Instead, researchers have turned to the use of dynamin inhibitors such as dynasore and dyngo-4a which allowed elucidating the role of dynamin in multiple forms of endocytosis that reportedly operate at the synapse (LinaresClemente et al., 2015; McCluskey et al., 2013; Watanabe et al., 2013). However, these dynamin inhibitors have been shown to also inhibit endocytosis in dynamin TKO fibroblasts suggesting that prior and future work with such inhibitors should be interpreted with caution (Park et al., 
61 2013). Moreover, off-target effects on other presynaptic pathways have been described (Douthitt

62 et al., 2011).

63 In this study, we show that synaptic vesicle recycling and neurotransmission in cultured

64 hippocampal neurons are unaffected by postnatal deletion of dynamin 2 indicating that this

65 dynamin isoform is not essential for synaptic function. Moreover, we were able to deplete all

66 three dynamin isoforms in cultures and found a decrease in the number of synaptic vesicles and

67 number of docked vesicles within the synapse suggesting a role for dynamins in synaptic

68 vesicle pool maintenance. More importantly, while the previously reported arrest in endocytosis

69 was observed after $20 \mathrm{~Hz}$ stimulation using the fluorescent reporter vGluT1-pHluorin, we show

70 that it occurs only in a fraction of all presynaptic boutons $(\sim 40 \%)$. The rest of synapses show

71 only an intermediate effect or the complete absence of it revealing the presence of dynamin-

72 independent endocytosis in central synapses. In line with this notion, we found that this

73 dynamin-independent recycling of individual synaptic vesicles, after either evoked or

74 spontaneous fusion, was largely impervious to disruption of actin, Arp2/3 and the dynamin-

75 related protein (DRP) function. Our results suggest that retrieval of synaptic vesicle proteins

76 does not require classical dynamin-dependent endocytic pathways after the release of single

77 synaptic vesicles. 


\section{Results}

\section{Dynamin 2 is not essential for synaptic vesicle recycling and neurotransmission}

Dynamin 2 catalyzes the scission of budding endosomes for various types of endocytosis apart from clathrin-mediated endocytosis (Cao et al., 2007; Liu et al., 2008; Schlunck et al., 2004) and it was reported to play a role in the exocytosis-endocytosis coupling of vesicles in mouse pancreatic $\beta$-cells (Min et al., 2007). In neurons, dynamin 2 has been reported to partially compensate for dynamin 1 knock out (KO) phenotype (Raimondi et al., 2011). Taken together, these findings hint at a potential role for dynamin 2 in synaptic vesicle recycling. Complete loss of dynamin 2 has proven to be too strenuous on developing tissue and as such, a complete dynamin $2 \mathrm{KO}$ mouse is non-viable in utero (Ferguson and De Camilli, 2012). Here, we successfully knocked out dynamin 2 postnatally in dissociated hippocampal neuron cultures using dynamin 2 floxed (dnm2f/f) mice and lentiviral delivery of the Cre recombinase. We confirmed dynamin 2 conditional knock out (Dnm2 cKO) by western blot analysis (Figure 1A). At 18 DIV, dynamin 2 levels were reduced by $(92.5 \pm 1.5) \%$ in neurons expressing the Cre recombinase (Dnm2 cKO) compared to control neurons (Figure 1A). Maximal depletion of dynamin 2 was observed after 17 DIV and thus all subsequent experiments were conducted at 17 DIV or later.

We next assessed synaptic vesicle recycling in the absence of dynamin 2 after high frequency stimulation using the optical indicator of synaptic vesicle exocytosis and endocytosis vGluT1pHluorin (Kavalali and Jorgensen, 2014; Leitz and Kavalali, 2011; Voglmaier et al., 2006). After a $20 \mathrm{~Hz} 100$ action potentials (APs) stimulation, there were no significant differences in the measured decay time constant ( $\mathrm{T}$ ) in Dnm2 cKO synapses ( 46 seconds) in comparison to WT littermate control synapses ( 49 seconds) (Figure 1B-C), indicating normal endocytic kinetics in neurons depleted of dynamin 2. The exocytic load was also unaltered in the absence of dynamin 2, since the ratio of the fluorescence amplitude $(\Delta \mathrm{F})$ after the $20 \mathrm{~Hz}$ stimulus to the maximal possible response obtained by perfusing ammonium chloride was similar in both groups (data not shown). We then investigated the retrieval of single synaptic vesicles after evoked exocytosis in Dnm2 cKO neurons by measuring the dwell time, the amount of time the pHluorin probe spent on the presynaptic membrane before being retrieved (Leitz and Kavalali, 2011; Chanaday and Kavalali, 2018). We observed no differences in dwell time in Dnm2 cKO neurons in comparison to the control suggesting that dynamin 2 is not required for retrieval after single synaptic vesicle release (Figure 1 - figure supplement $1 A-B$ ). In addition, amplitude of fusion events (Figure 1 - figure supplement 1C) and release probability (data not shown) were 
114 similar for control and Dnm2 cKO neurons. Finally, we assessed the effects of the loss of

115 dynamin 2 on synaptic transmission by voltage patch clamp recordings and observed no

116 changes in average evoked and spontaneous excitatory (eEPSC and mEPSC, respectively)

117 activity implying that dynamin 2 is not essential for synaptic transmission (Figure 1D-G and

118 Figure 1 - figure supplement 1D-H). Taken together, these results suggest that dynamin 2 is not

119 critical for synaptic vesicle recycling or neurotransmission.

\section{Synapse morphology in the absence of dynamins 1, 2 and 3}

121 Previous findings suggested the possibility of dynamin-independent endocytic mechanisms in 122 neurons (Xu et al., 2008). To explore the role of all three dynamin isoforms in synaptic vesicle 123 recycling, we crossed a knock-out $(\mathrm{KO})$ mouse line for dynamin 3 and mice with floxed dynamin

1241 and 2 genes. After Cre recombinase treatment, triple knock-out (TKO) neurons for all dynamin 125 isoforms were obtained (Dnm TKO). We used hippocampal neuron cultures from littermate 126 animals infected with the empty vector (expressing only GFP) as controls (Dnm3 KO). We used

127 Dnm3 KOs as controls in order to obtain more consistent comparisons within littermates based

128 on the observation that Dnm3 KOs do not show significant functional or structural deficits

129 (Ferguson et al., 2007; Raimondi et al., 2011). To confirm that all 3 dynamin isoforms were

130 knocked out we measured protein levels by Western blot (using a pan-dynamin antibody; Liu et

131 al., 2008) and mRNA expression by q-RT PCR. While protein levels of all dynamins were

132 reduced by $(92.9 \pm 0.4) \%$ (Figure $1 \mathrm{H}$ ), mRNA levels of dynamins 1 and 2 were reduced $>99 \%$

133 while dynamin 3 mRNA was reduced $>95 \%$ in Dnm TKO neurons (Figure 1 - figure supplement

$1342 \mathrm{~A}$ ), indicating that all dynamins are significantly depleted under these conditions. We then

135 analyzed synapse morphology using electron microscopy (EM) in Dnm TKO neurons and

136 observed no gross distortions in synapse morphology at rest. However, we found a significant

137 decrease in the total synaptic vesicle number ( 65 vs 44) and the number of docked vesicles

138 ( 3 vs 1.5) in Dnm TKO neurons in comparison to Dnm3 KO littermate control neurons (Figure

139 1I-K). Given that neurons and synapses are only starting to develop and mature at the time of

140 lentiviral infection, we next evaluated the effects of loss of dynamins on synapse numbers. We

141 labeled Dnm TKO and control cultures for inhibitory synapses using an antibody against the

142 vesicular GABA transporter (vGAT) and excitatory synapses using an antibody against the

143 vesicular glutamate transporter (vGluT). We observed no significant differences in the total

144 number of excitatory and inhibitory synapses per $\mu \mathrm{m}^{2}$ in Dnm TKO cultures compared to

145 littermate controls (Figure 1L-N). This data suggests that dynamin is required for the

146 maintenance of synaptic vesicle pool size and the docking of synaptic vesicles at rest but not for 
synapse formation.

148 Synaptic vesicle recycling during high frequency stimulation in neurons depleted of 149 dynamins $1,2,3$

150 Next, we assessed synaptic vesicle endocytosis in response to 100 APs at $20 \mathrm{~Hz}(5 \mathrm{~s})$ by 151 expressing vGluT1-pHluorin in Dnm TKO neurons (Figure 2A). We observed three categorically

152 different responses to the $20 \mathrm{~Hz}$ stimulus in neurons depleted of dynamins: synapses that

153 recovered completely after $20 \mathrm{~Hz}$ stimulation, synapses that recovered to a baseline higher than

154 the baseline prior to stimulation (partial recovery) and those that did not recover after stimulation

155 (Figure $2 \mathrm{~B})$. To quantify this phenotype, we first calculated the amount of retrieval $\left(\Delta \mathrm{F}_{\text {ret }}\right)$ as the

156 difference between baseline level increase after stimulation and response amplitude $(\Delta \mathrm{F})$, and

157 then obtained the percentage of retrieval by dividing $\Delta \mathrm{F}_{\text {ret }}$ by $\Delta \mathrm{F}$. We observed that in Dnm3 KO

158 littermate controls, $90 \%$ of synapses recovered to $80 \%$ of the initial baseline within $150 \mathrm{~s}$ after

159 stimulation, while around $60 \%$ of synapses recovered to at least $80 \%$ of the initial baseline

160 within $150 \mathrm{~s}$ post stimulus in Dnm TKO neurons. This suggests that even if all three dynamin

161 isoforms are significantly reduced, synaptic vesicles can still undergo normal endocytosis in

162 more than half of hippocampal synapses (Figure 2B-C). We observed a decrease in the ratio of

$163 \Delta \mathrm{F}$ to the maximal possible response, $\mathrm{F}_{\mathrm{NH} 4 \mathrm{Cl}}\left(\Delta \mathrm{F} / \mathrm{F}_{\mathrm{NH} 4 \mathrm{Cl}}\right)$ in Dnm TKO compared to control

164 (Figure 2D) suggestive of a decrease in release probability in the absence of all dynamins and corroborating our finding of decreased number of docked vesicles via EM (Figure $1 \mathrm{~K}$ ). We also detected an overall decrease in $\mathrm{F}_{\mathrm{NH} 4 \mathrm{Cl}}$ in Dnm TKO neurons in comparison to control suggesting a possible decrease in synaptic vesicle pool size as well (data not shown, but see Figure 1J). Finally, we estimated the kinetics of endocytosis by fitting the decay to baseline after stimulus with a single exponential decay function and used the decay time constant $(\mathrm{T})$ as a measure of endocytic rate. We observed a drastic increase in decay $\mathrm{T}$ in Dnm TKO neurons indicating a slow-down in synaptic vesicle retrieval after high frequency stimulation (Figure 2E). Taken

172 together, these results suggest that dynamins are important but not essential for synaptic

173 vesicle recycling during high frequency neuronal activity.

174 Given our observation of almost normal synaptic vesicle endocytosis for $\sim 60 \%$ of synapses after

$17520 \mathrm{~Hz}$ stimulation, we next asked whether the retrieved vesicles are available for re-release. We 176 stimulated synaptic uptake of FM5-95 dye by incubating Dnm TKO and littermate control

177 neurons in $45 \mathrm{mM} \mathrm{K}^{+}$modified Tyrode's solution containing $18 \mu \mathrm{M}$ FM5-95 dye for 2 minutes.

178 Excess dye was washed out, and then we applied a second stimulation to trigger synaptic

179 vesicle exocytosis and dye release by incubating neuronal cultures in $90 \mathrm{mM} \mathrm{K}^{+}$modified 
180 Tyrode's solution for $90 \mathrm{~s}$, two consecutive times (Figure 2F). We observed a decrease in

181 fluorescence indicative of dye release in both littermate control and Dnm TKO synapses (Figure

$1823 \mathrm{~F})$. However, the decrease in fluorescence in response to $90 \mathrm{mM} \mathrm{K}^{+}$stimulation was greater in

183 littermate controls than in Dnm TKO (Figure 2G) and it also occurred with a slower time-course

184 in Dnm TKO synapses (Figure 2H). These results suggest that depletion of dynamins reduces

185 the total pool size of synaptic vesicles and the remaining vesicles show slower mobilization and

186 recovery compared to controls during strong depolarization. Our findings are also consistent

187 with a function for dynamins in the regulation of synaptic vesicle release probability (Lou et al.,

1882012 and see below).

\section{Single synaptic vesicle endocytosis is dynamin independent}

190 So far, we have described dynamin function in synaptic vesicle recycling during strong

191 stimulation. To address the importance of dynamins in the recycling of individual synaptic

192 vesicles, we applied sparse, single AP stimulation to Dnm TKO hippocampal neuron cultures

193 expressing vGluT1-pHluorin. We observed no change in the amplitude of single synaptic vesicle

194 fusion events in Dnm TKO synapses in comparison to littermate control Dnm3 KO synapses

195 (Figure 2I-K). No differences were observed in the distribution of dwell times in presynaptic

196 boutons from both groups (Figure 2L). Moreover, exponential fitting of the ensemble average

197 traces from Dnm3 KO and TKO groups revealed the presence of two phases of decay, one

198 ultrafast ( $300 \mathrm{~ms}$ ) and one fast ( 6-8 s) of similar characteristics for both groups (Figure 2l-J)

199 (see Chanaday and Kavalali, 2018). This further supports the absence of defects in retrieval and

200 re-acidification in dynamin TKO neurons compared to Dnm3 KO. Finally, we categorized the

201 single synaptic vesicle fusion events into three groups based on the event profile (see sample

202 traces in Figure 2l-J). Rapid decay were events that decayed almost instantaneously after

203 fusion ( $<500 \mathrm{~ms}$ ) and may correspond to ultrafast endocytosis events. Short dwell were events

204 that resided on the membrane for more than $500 \mathrm{~ms}$ and decayed to baseline before the end of

205 the allotted timeframe (500 ms - $10 \mathrm{~s}$ ). Long dwell were events that did not decay to baseline

206 within the allotted time window (> $10 \mathrm{~s}$ ). We measured the fractional composition of the single

207 synaptic vesicle fusion events in these three categories and noted no changes in the distribution

208 between Dnm TKO and littermate control Dnm 3 KO synapses (Figure 2M). Long dwell events,

209 which are indicative of protein stranded at the plasma membrane and not retrieved during the

210 imaging window, constituted $19 \pm 1 \%$ of total dynamin TKO single vesicle events in comparison

211 to $21 \pm 1 \%$ of total control single vesicle events (Figure $2 \mathrm{M}$ ). We also looked at the fractional

212 composition of single synaptic vesicle events from Dnm TKO synapses with severe retardation 
213 of synaptic vesicle endocytosis after high frequency stimulation and observed that only $\sim 1 \%$ of

214 single vesicle events from these synapses were long-dwell events. This observation suggests

215 that the negative impact of dynamin loss-of-function in these synapses was limited to multi-

216 vesicle endocytosis and the dynamin-regulation of single vesicle versus multi-vesicle retrieval

217 events are relatively independent (Figure 2M). We assessed the distribution of rapid decay and

218 short dwell events and observed no differences between Dnm $3 \mathrm{KO}$ and Dnm TKO (Figure 2M).

219 Taken together, our data suggest that the kinetic properties of single synaptic vesicle retrieval

220 are largely dynamin independent.

\section{Dynamins are not essential for $\mathrm{Ca}^{2+}$ dependent delay in single synaptic vesicle retrieval}

222 Prior work in our lab has demonstrated that increasing extracellular $\mathrm{Ca}^{2+}$ can slow down

223 endocytosis after single synaptic vesicle release (Leitz and Kavalali, 2011). This retardation in

224 synaptic vesicle retrieval is dependent on synaptotagmin-1 (syt1), the $\mathrm{Ca}^{2+}$ sensor for

225 synchronous synaptic vesicle exocytosis (Li et al., 2017; Chanaday and Kavalali, 2018). In

226 chromaffin cells, syt1 has been shown to interact directly with the PH domain of dynamin 1

227 (Dnm1) to regulate the fission pore of single dense core vesicles (McAdam et al., 2015). To

228 address the role of dynamins in the modulation of synaptic vesicle retrieval kinetics by $\mathrm{Ca}^{2+}$, we

229 increased extracellular $\mathrm{Ca}^{2+}$ concentration to $8 \mathrm{mM}$ and evoked single synaptic vesicle release

230 in Dnm TKO and littermate control neurons (Figure 3A-B). We observed no differences in the

231 amplitude of single synaptic vesicle fusion events in these conditions (Figure $3 \mathrm{C}$ ). The

232 distribution of dwell times (Figure 3D) as well as the two components of fluorescence decay for

233 the average traces (Figure 3A-B) were similar for Dnm3 KO and Dnm TKO synapses. This

234 result indicates that $\mathrm{Ca}^{2+}$ can slow down single synaptic vesicle endocytosis in synapses lacking

235 all dynamin isoforms, as described before for wild-type neurons (Leitz and Kavalali, 2011; Li et

236 al., 2017) leading us to conclude that dynamins are not essential for $\mathrm{Ca}^{2+}$-dependent

237 prolongation of single synaptic vesicle dwell time. Moreover, based on the analysis of the

238 ensemble averaged fluorescent traces, the kinetics of ultrafast endocytosis and fast endocytosis

239 components of retrieval do not seem to be modulated by dynamins (Chanaday and Kavalali,

240 2018).

241 Inhibitory neurotransmission after depletion of dynamins

242 Our data suggests that the postnatal deletion of dynamin results in a decreased pool size of

243 synaptic vesicles that can recycle with sparse stimulation but are limited upon high frequency

244 stimulation. Prior work has demonstrated that the efficiency of synaptic vesicle endocytosis is

245 dependent on the preceding exocytic load (Armbruster et al., 2013). Inhibitory synapses have 
been reported to undergo tonic neurotransmission and as such require more efficient endocytic mechanisms to maintain fidelity of neurotransmission (Evergren et al., 2006; Swadlow, 2003). Therefore, synaptic vesicle recycling in non-glutamatergic synapses might be different from glutamatergic synapses. To assess the dynamin dependence of inhibitory transmission, we recorded evoked inhibitory postsynaptic currents (eIPSC) in Dnm TKO neurons (Figure 4). We observed a decrease in the average elPSC amplitude (0.8 nA vs $1.3 \mathrm{nA})$ in Dnm TKO neurons in comparison to the littermate controls (Figure 4A). We applied a train of 30 pulses at $1 \mathrm{~Hz}$ and observed similar levels of synaptic depression in Dnm TKO and littermate control cultures (Figure 4B-C). Similarly, we observed similar levels of synaptic depression after we challenged Dnm TKO and littermate control neurons with a 400 pulse $10 \mathrm{~Hz}$ train stimulus (Figure 4D-E). However, when we stimulated with 1000 pulses at $30 \mathrm{~Hz}$ frequency, we observed an initial synaptic facilitation in Dnm TKO neurons that was absent in littermate control Dnm3 KO neurons. This data is consistent with the premise that the absence of dynamin in inhibitory neurons leads to a decrease in release probability. We also investigated spontaneous or miniature inhibitory neurotransmission (mIPSC) in the presence of tetrodotoxin (TTX). We observed no significant changes in the frequency of mIPSC events between groups $(1.8 \mathrm{~Hz}$ vs $1.4 \mathrm{~Hz}$ ) but found a rightward shift in the cumulative distribution of amplitudes for Dnm TKO neurons indicative of an overall increase in mIPSC amplitude (Figure 4G-I). Since no alterations in synaptic vesicle size were found by EM (data not shown), this could indicate differences in postsynaptic levels of GABA receptors. In conclusion, for inhibitory synapses, depletion of all three dynamin isoforms leads to a reduction of evoked release probability and an increase in amplitude of spontaneous postsynaptic responses.

\section{Loss of dynamins 1, 2 and 3 impairs excitatory neurotransmission}

Excitatory neurotransmission was previously shown to have reduced release probability and a decrease in the amplitude of postsynaptic currents in the absence of the neuronal dynamin isoforms, dynamin 1 and 3. These alterations could be reversed by chronic suppression of activity (Lou et al., 2012) indicating that it results from vesicle depletion due to the endocytic arrest. To evaluate excitatory neurotransmission when all dynamin isoforms are depleted, we measured evoked excitatory postsynaptic currents (eEPSC) in Dnm TKO hippocampal neurons (Figure 5). We observed a significant decrease in eEPSC amplitude (1.1 nA vs $0.1 \mathrm{nA}$ ) in Dnm TKO neurons (Figure 5A) accompanied by facilitation to a train of $1 \mathrm{~Hz}$ and $10 \mathrm{~Hz}$ stimuli (Figure $5 \mathrm{~B}-\mathrm{E})$ suggestive of a decrease in the probability of release in Dnm TKO neurons compared to Dnm3 KO littermate control. We also investigated changes in spontaneous neurotransmission in 
the presence of TTX in Dnm TKO neurons. We observed a decrease in mEPSC frequency (1.7 $\mathrm{Hz}$ vs $0.7 \mathrm{~Hz}$ ) and an increase in mEPSC amplitude (Figure 5F-H) in Dnm TKO neurons compared to control. These findings are consistent with prior reports assessing excitatory neurotransmission defects in constitutive dynamin 1,3 double KO neurons (Lou et al., 2012) pointing to a negligible role for dynamin 2 in excitatory neurotransmission.

\section{Spontaneously fused synaptic vesicles recycle independently of actin, DRP-1 and Arp2/3} complex in dynamin TKO neurons

Our results so far have revealed that dynamin is not required for the recycling of a single synaptic vesicle after either evoked or spontaneous fusion. We next attempted to identify crucial proteins for the recycling of a single synaptic vesicle in Dnm TKO neurons. In multiple cell types, dynamin is localized to Arp2/3 complex enucleated actin meshwork (Baldassarre et al., 2003; Gold et al., 1999; Lee and De Camilli, 2002; Orth et al., 2002; Schlunck et al., 2004). Similarly, during clathrin-mediated endocytosis, both actin and dynamin are recruited to the site of retrieval in the early phase suggesting a functional significance in initiation and maturation of clathrin-coated pits (Grassart et al., 2014). In both the large calyx of Held and small central hippocampal synapses, knockout of actin isoforms revealed that actin functions in all forms of synaptic vesicle endocytosis (Wu et al., 2016). Here, we explored the role of actin and Arp2/3 complex in the recycling of single synaptic vesicles by using the small molecule inhibitors Latrunculin A and CK-666 to inhibit actin and Arp2/3 complex, respectively. We also assessed dynamin-related protein 1 (DRP-1) function in synaptic vesicle recycling using the specific inhibitor Mdivi-1, as previous studies described that mutations in DRP in Drosophila leads to synaptic vesicle depletion (Rikhy et al., 2007). We knocked out all isoforms of dynamin in cultured hippocampal neurons and recorded mEPSC events before and after acute (10 $\mathrm{min})$ treatment with each small molecule inhibitor (Figure 6). These experiments did not reveal significant differences in mEPSC frequency after treatment with Latrunculin A, CK-666 or Mdivi1 (Figure 6A-B). Taken together, our findings suggest that dynamin, actin, arp2/3 complex and DRP-1 do not play a major role in the recycling of single spontaneously released synaptic vesicles. 


\section{Discussion}

In this study, to investigate the functional diversity of dynamins, we established a colony of dynamin mutant mice comprised of dynamin-1 and dynamin-2 conditional knockouts (expressing floxed alleles of dynamin-1 and -2), and dynamin-3 constitutive knockouts. Using conditional knockouts to suppress dynamin expression in vitro, we could avoid systemic effects and impair dynamin function after synaptogenesis thus circumventing major developmental effects. When we assessed dynamin-2 loss-of-function in synaptic vesicle recycling and neurotransmission, we did not observe any apparent defects after the postnatal deletion of dynamin-2. In contrast, depletion of all three dynamins (dynamin-1, -2 and -3) in hippocampal neurons in culture impaired neurotransmission, including a decrease in release probability and also a substantially slowed endocytosis after high frequency stimulation $(20 \mathrm{~Hz})$. These effects were more pronounced in recordings of glutamatergic synaptic transmission compared to GABAergic neurotransmission suggesting a potential difference in dynamin-dependence between the two neurotransmitter systems. We also visualized dynamin TKO synapses via electron microscopy and observed decreases in both synaptic vesicle number and number of docked vesicles, which may in part account for the decrease in evoked neurotransmitter release probability.

Despite the apparent decrease in evoked release, the frequency of spontaneous neurotransmitter release events (as detected by postsynaptic voltage-clamp recordings) and the kinetics of single vesicle fusion-retrieval events (as detected optically) were only mildly affected. This surprising result is, nevertheless, consistent with earlier findings based on alternative methods from our group as well as others. For instance, treating neurons with the small molecule inhibitor of dynamin, dynasore, capable of inhibiting endocytosis, did not elicit any defects in spontaneous neurotransmission although evoked synchronous and asynchronous transmission showed significant activity-dependent suppression (Chung et al., 2010). Similarly, the injection of non-hydrolyzable GTP into the calyx of Held synapse revealed an initial block of synaptic vesicle retrieval that was followed by a resumption of synaptic vesicle endocytosis (Xu et al., 2008). In addition, a study in salamander retinal cone cells, a tonically active synapse, revealed no change in endocytosis in the presence of dynamin inhibitors (Van Hook and Thoreson, 2012). These reports suggest the presence of dynamin-independent endocytosis mechanisms in neurons contributing to the retrieval of synaptic vesicles from the presynaptic plasma membrane.

Our results suggest that dynamins play a key role in regulation of evoked probability of release 
and the retrieval of synaptic vesicle components after strong stimulation. In addition to the decrease in synaptic vesicle numbers, decreased release probability can be a consequence of limited availability of release sites due to imperfect clearance of fused vesicles during sustained high frequency activity (Kawasaki et al., 2000; Lou et al., 2012; Hua et al., 2013). Given the relative sparsity of spontaneous fusion events, the impairments in clearance of release sites may not present a major impediment for spontaneous fusion propensity. However, it is also important to note that in the absence of dynamins we did not detect a major impairment in the kinetic properties of retrieval after single synaptic vesicle fusion events indicating that the maintenance of spontaneous fusion events cannot solely be explained by their low frequency, but rather suggest a role for dynamin-independent recycling mechanisms.

In this study, we used lentiviral delivery of Cre-recombinase to delete dynamin-1 and dynamin-2 expression on the background of dynamin-3 constitutive knock out neurons. This approach resulted in swift depletion of dynamins as detected by Western blots as well as quantitative RTPCR analysis. This substantial depletion of all dynamin isoforms is consistent with earlier measurements of dynamin protein lifetime in neurons (Fornasiero et al., 2018). However, we cannot fully exclude the possibility that some residual dynamins, below our detection, may contribute to the intact synaptic vesicle trafficking events we detect under these conditions. Such an effect of residual dynamins would nevertheless suggest a steep functional selectivity of dynamins depending on their abundance. According to this premise, while ultralow dynamin levels would be sufficient to maintain quantal synaptic vesicle trafficking, majority of activity driven synaptic vesicle endocytosis requires high levels of dynamin expression.

These results open new critical questions. What are the possible mechanisms that activate this putative dynamin-independent endocytosis that appears to be specific to single synaptic vesicle retrieval and spontaneous neurotransmission? How does membrane scission operate without the perennial pinchase dynamin? Although we do not have answers to these questions at this time, our findings highlight some possibilities. Our findings using small molecule inhibitors suggest that actin is not a key player for maintenance of quantal neurotransmission when dynamins are depleted. Recent studies focusing on the uptake of bacterial Shiga and cholera toxins, revealed that clathrin-independent endocytic events - that are often less reliant on dynamin - actually utilize BAR domain proteins such as endophilins for membrane scission (Renard et al., 2015). Therefore, we cannot exclude the possibility that endophilins such as endophilin-A2 and the synapse enriched endophilin-A1 (e.g. Llobet et al., 2011) may play critical roles in dynamin-independent synaptic vesicle retrieval. Overall, future experiments aimed at 
bioRxiv preprint doi: https://doi.org/10.1101/2020.06.12147975; this version posted June 12, 2020. The copyright holder for this preprint (which was not certified by peer review) is the author/funder, who has granted bioRxiv a license to display the preprint in perpetuity. It is made available under aCC-BY 4.0 International license.

374 uncovering the molecular mechanism of single synaptic vesicle retrieval will also help elucidate 375 the physiological role of this dynamin-independent quantal neurotransmission.

376 


\section{Materials and Methods.}

379 KEY RESOURCES TABLE

\begin{tabular}{|c|c|c|}
\hline REAGENT or RESOURCE & SOURCE & IDENTIFIER \\
\hline \multicolumn{3}{|l|}{ Antibodies } \\
\hline Mouse monoclonal anti-Dynamin 1 & Abcam & Ab13251 \\
\hline Rabbit polyclonal anti-Dynamin 2 & Abcam & Ab3457 \\
\hline Rabbit polyclonal anti-pan Dynamin & Liu et al., 2008 & $\mathrm{~N} / \mathrm{A}$ \\
\hline Mouse monoclonal anti-Rab-GDI1 & Synaptic Systems & 130011 \\
\hline Mouse monoclonal anti-Synapsin & Synaptic Systems & 106001 \\
\hline Mouse monoclonal anti-vGluT & Synaptic Systems & 135511 \\
\hline Mouse monoclonal anti-vGAT & Synaptic Systems & 131011 \\
\hline \multicolumn{3}{|l|}{ Chemicals, Peptides, and Recombinant Proteins } \\
\hline $\begin{array}{l}\text { 6-Cyano-7-nitroquinoxaline-2,3-dione disodium salt } \\
\text { hydrate (CNQX) }\end{array}$ & Sigma-Aldrich & Catalog \# C239 \\
\hline D(-)-2-Amino-5-phosphonopentanoic acid (AP-5) & Sigma-Aldrich & Catalog \# A8054 \\
\hline Picrotoxin (PTX) & Sigma-Aldrich & Catalog \# P1675 \\
\hline Tetrodotoxin (TTX) & $\begin{array}{l}\text { Enzo Life } \\
\text { Sciences }\end{array}$ & $\begin{array}{l}\text { Catalog \# BML-NA120- } \\
0001\end{array}$ \\
\hline Latrunculin-A & Tocris & Catalog \# 3973 \\
\hline CK-666 & Tocris & Catalog \# 3950 \\
\hline Mdivi-1 & Sigma-Aldrich & Catalog \# M0199 \\
\hline FM5-95 & Molecular Probes & Catalog \# T23360 \\
\hline \multicolumn{3}{|l|}{ Experimental Models: Cell Lines } \\
\hline Human embryonic kidney-293 (HEK-293) cells & ATCC & Catalog \# CRL-1573 \\
\hline \multicolumn{3}{|l|}{ Experimental Models: Organisms/Strains } \\
\hline Jackson Laboratory fDnm1 Mice & $\begin{array}{l}\text { Jackson } \\
\text { Laboratory }\end{array}$ & 013073 \\
\hline Jackson Laboratory fDnm2 Mice (P0-P3) & $\begin{array}{l}\text { Jackson } \\
\text { Laboratory }\end{array}$ & 013542 \\
\hline Jackson Laboratory Dnm3 Mice & $\begin{array}{l}\text { Jackson } \\
\text { Laboratory }\end{array}$ & 013543 \\
\hline \multicolumn{3}{|l|}{ Recombinant DNA } \\
\hline Plasmid: pRSV-REV (lentiviral packaging) & Adgene & Catalog \# 12253 \\
\hline Plasmid: pCMV-VSV-G (lentiviral packaging) & Adgene & Catalog \# 8454 \\
\hline Plasmid: pMDLg/pRRE (lentiviral packaging) & Adgene & Catalog \# 12251 \\
\hline Plasmid: pFUGW-vGlut1-pHGFP & $\begin{array}{l}\text { Voglmaier et al., } \\
2006\end{array}$ & $\mathrm{~N} / \mathrm{A}$ \\
\hline Plasmid: L307 & & $\mathrm{N} / \mathrm{A}$ \\
\hline Plasmid: Cre recombinase-GFP & & $\mathrm{N} / \mathrm{A}$ \\
\hline Plasmid: pFUGW-vGlut1-pHGFP-Cre recombinase & & $\mathrm{N} / \mathrm{A}$ \\
\hline \multicolumn{3}{|l|}{ Sequence-Based Reagents } \\
\hline CTACCACAGAATATGCCGAGT & Dnm1 forward & This paper \\
\hline ACTGGGACCTTGGTCATTCC & Dnm1 reverse & This paper \\
\hline TCACACCTGCCAACATGGAC & Dnm2 forward & This paper \\
\hline CGCCGATATAGCCTCTTCTCA & Dnm2 reverse & This paper \\
\hline GAATTTCGTGGGCAGGGACT & Dnm3 forward & This paper \\
\hline
\end{tabular}


380

381

382 ETHICS

383 media.

\begin{tabular}{|l|l|l|}
\hline TTACGCGGTCTGTTTCTGCT & Dnm3 reverse & This paper \\
\hline \multicolumn{2}{|l|}{} & \\
\hline \multicolumn{2}{|l|}{} \\
\hline Software and Algorithms & \\
\hline Matlab script for pHluorin analysis & Chanaday et al., & N/A \\
& 2018 & \\
\hline Fiji & Schindelin et al., & N/A \\
& 2012 & \\
\hline
\end{tabular}

Animal experimentation: Animal procedures conformed to the Guide for the Care and Use of Laboratory Animals and were approved by the Institutional Animal Care and Use Committee at UT Southwestern Medical Center (Animal Protocol Number APN 2016-101416) and at Vanderbilt University School of Medicine (Animal Protocol Number M1800103).

\section{METHOD DETAILS}

\section{Lentiviral infection}

HEK293 cells (ATCC) were transfected with 3 lentiviral packaging plasmids (pMDLg/pRRE, pRSV-Rev, and pCMV-VSV-G) and a pFUGW vector containing a Crerecombinase and/or vGluT1-pHluorin construct using the Fugene 6 transfection reagent (Promega). Cell culture supernatants containing the virus were collected 72 hours later and spun down to precipitate out cellular debris and other contaminants. Neurons were infected at 4 days in vitro (DIV) by adding $200 \mu$ l of virus containing supernatant to the neuronal culture

\section{Cell culture}

Dissociated hippocampal cultures from postnatal day 0-3 dnm $1^{\mathrm{flf}} \mathrm{dnm} 2^{\mathrm{f} / \mathrm{f}} \mathrm{dnm} 3 \mathrm{KO}$ were prepared as previously described (Kavalali, Klingauf, \& Tsien, 1999). Neurons were infected at 4 DIV with lentivirus expressing Cre recombinase or an empty L307 vector for control and experiments were performed at 17-21 DIV. All experiments were performed following protocols approved by the University of Texas Southwestern Institutional Animal Care and Use Committee.

\section{Immunocytochemistry}

Neuronal cultures were processed for immunocytochemistry as described in Ramirez et. 
al. (2008) at 17-19 DIV. Antibodies against dynamin (Synaptic Systems), dynamin 1 (Abcam), dynamin 2 (Abcam), synapsin, vGlut-1 (Synaptic Systems) and vGAT (Synaptic Systems) were used at concentrations of 1:500,1:300,1:300,1:1000, 1:500 and 1:500 respectively. Images were taken on a confocal microscope with a 60X 1.4 NA objective. 8-10 images were taken from 3 coverslips per group. Colocalization analysis was object based and performed using Fiji software (Schindelin et al. 2012) and a custom made macro.

\section{Electron Microscopy}

Neuronal cultures were incubated in modified Tyrode's buffer $(145 \mathrm{mM} \mathrm{NaCl}, 4 \mathrm{mM} \mathrm{KCl}, 2$ $\mathrm{mM} \mathrm{MgCl}$, $10 \mathrm{mM}$ glucose, $10 \mathrm{mM}$ HEPES, $2 \mathrm{mM} \mathrm{CaCl}_{2}, \mathrm{pH}$ 7.4, osmolarity $310 \mathrm{mOsM}$ ) for 2 minutes and then rinsed twice with PBS and then fixed and processed for electron microscopy by the UTSW Electron Microscopy Core.

\section{Western Blot}

Neuronal cultures were homogenized processed for western blot as described in Nosyreva and Kavalali (2010). Antibodies against dynamin (Liu et al., 2008), dynamin 1 (Abcam) and dynamin 2 (Abcam) were used at a 1:1000, 1:3000 and 1:800 dilution respectively and protein bands were developed using enhanced chemiluminescence (ECL). Bands were analyzed using ImageJ software and protein levels were normalized to GDI loading control.

\section{RNA extraction and quantitative-Reverse Transcriptase (qRT) PCR}

To determine relative expression of different dynamin mRNA isoforms after lentivirus infection, we performed qRT-PCR. RNA from the DIV 19 neuronal cultures were collected by using PureLink RNA Mini Kit (Ambion, Cat. \# 12183018A). Quality and purity of RNA were confirmed by NanoDrop2000. For cDNA synthesis, we used Invitrogen's SuperScript TM III Reverse Transcriptase (Cat. \#18080-093). Following its first-strand cDNA synthesis protocol, we used Promega's Oligo(dT)15 (Cat. \# C1101) primer and Recombinant RNasin® Ribonuclease Inhibitor (N2511). cDNA concentration and quality were confirmed by NanoDrop2000 before qRT PCR.

By following Appliedbiosystems PowerUp ${ }^{\mathrm{TM}}$ SYBR $^{\mathrm{TM}}$ Green Master Mix (Cat. \#A25742) protocol, transcripts for Dnm1, Dnm2, Dnm3 and Gapdh were amplified in a Stratagene Mx3005P. Thermal cycling conditions consisted of 1 cycle of $50{ }^{\circ} \mathrm{C}$ for $2 \mathrm{~m}$ and $95{ }^{\circ} \mathrm{C}$ for $2 \mathrm{~m}, 40$ cycles of $95^{\circ} \mathrm{C}$ for $15 \mathrm{~s}, 57^{\circ} \mathrm{C}$ for $45 \mathrm{~s}, 72^{\circ} \mathrm{C}$ for $60 \mathrm{sec}$, and 1 dissociation cycle of $95^{\circ} \mathrm{C}$ for 15 $\mathrm{s}, 60{ }^{\circ} \mathrm{C}$ for $60 \mathrm{~s}, 95^{\circ} \mathrm{C}$ for $15 \mathrm{~s}$. Respective primers were obtained from Integrated DNA Technologies: 5'-CTACCACAGAATATGCCGAGT-3' and 5'-ACTGGGACCTTGGTCATTCC-3' for 
Dnm1; 5'-TCACACCTGCCAACATGGAC-3' and 5'-CGCCGATATAGCCTCTTCTCA-3' for Dnm2; 5'-GAATTTCGTGGGCAGGGACT-3 and 5'-TTACGCGGTCTGTTTCTGCT-3' for Dnm3; 5'-AGG TCG GTG TGA ACG GAT TTG-3' and 5'-TGT AGA CCA TGT AGT TGA GGT CA-3' for Gapdh. We calculated relative quantification by using $2-\Delta \Delta C$ t. The number 2 in the equation indicates

442 DNA doubling in each cycle.

\section{Electrophysiology}

A modified Tyrode's solution was used for all experiments (except where noted otherwise). Pyramidal neurons were whole-cell voltage clamped at $-70 \mathrm{mV}$ with borosilicate glass electrodes (3-5 M 2 ) filled with a solution containing (in $\mathrm{mM}$ ): 105 Cs-methanesulphonate, $10 \mathrm{CsCl}, 5 \mathrm{NaCl}, 10$ HEPES, 20 TEA.Cl hydrate, 4 Mg-ATP, 0.3 GTP, 0.6 EGTA, 10 QX-314 (pH 7.3, osmolarity $300 \mathrm{mOsM}$ ). Excitatory-postsynaptic currents (EPSCs) were evoked with 0.1 ms, $10 \mathrm{~mA}$ pulses delivered via a bipolar platinum electrode in a modified Tyrode's solution containing Picrotoxin (PTX, $50 \mu \mathrm{M})$ and D-2-Amino-5-phosphonovaleric acid (D-APV, $50 \mu \mathrm{M}$, NMDA receptor blocker). Spontaneous miniature EPSCs (mEPSCs) were recorded in a modified Tyrode's solution containing TTX $(1 \mu \mathrm{M})$, PTX $(50 \mu \mathrm{M})$ and D-APV $(50 \mu \mathrm{M})$. Inhibitorypostsynaptic currents (IPSCs) were evoked with $0.1 \mathrm{~ms}, 10 \mathrm{~mA}$ pulses delivered via a bipolar platinum electrode in a modified Tyrode's solution containing 6-Cyano-7-nitroquinoxaline-2-3dione (CNQX, $10 \mu \mathrm{M}$, AMPA receptor blocker) and D-APV $(50 \mu \mathrm{M})$. Spontaneous miniature IPSCs (mIPSCs) were recorded in a modified Tyrode's solution containing TTX $(1 \mu \mathrm{M}), \mathrm{CNQX}$ $(10 \mu \mathrm{M})$ and D-APV $(50 \mu \mathrm{M})$. Data was analyzed offline with Clampfit 9 software.

\section{Imaging}

vGlut1-pHluorin: 17-21 DIV neuronal cultures infected with lentivirus expressing either vGluT1-pHluorin or vGluT1-pHluorin and Cre recombinase were used for imaging experiments. Images were taken with an Andor iXon Ultra 897 High Speed Camera (Andor Technology Ltd) through a Nikon Eclipse TE2000-U Microscope (Nikon) using a 100X Plan Fluor objective (Nikon). Images were illuminated with a Lambda-DG4 (Sutter instruments) and acquired at $\sim 7$ $\mathrm{Hz}$ with a $120 \mathrm{~ms}$ exposure time and binned at 4 by 4 to increase the signal-to-noise ratio. Images were collected and processed using Nikon Elements Ar software prior to export to Microsoft excel for analysis. ROls were randomly selected based on a threshold after treatment with $\mathrm{NH}_{4} \mathrm{Cl}$.

FM5-95 dye: 17-21 DIV hippocampal neuron cultures infected with lentivirus expressing either Cre-recombinase and GFP or an empty L307 vector were used. Cultures were incubated 
for 2 minutes in a $45 \mathrm{mM} \mathrm{K}^{+}$modified tyrode's solution containing FM5-95 dye at a concentration of $18 \mu \mathrm{M}$. Subsequently, excess dye not endocytosed was washed out for 7 minutes with $2 \mathrm{mM}$ $\mathrm{Ca}^{2+}$ tyrode's solution and synaptic vesicle release of dye was stimulated by two perfusions of $90 \mathrm{mM} \mathrm{K}^{+}$modified tyrode's solution for 90 minutes each separated by a 90 min washing with 2 $\mathrm{mM} \mathrm{Ca}^{2+}$ tyrode solution. Images were captured with a Nikon Eclipse TE2000-U Microscope (Nikon) using a 40X Fluor objective (Nikon). Images were illuminated with a Lambda-DG4 (Sutter instruments) and acquired at $1 \mathrm{~Hz}$ with a $200 \mathrm{~ms}$ exposure time. Images were collected and processed using Nikon Elements Ar software prior to export to Microsoft excel for analysis. ROls were randomly selected.

\section{Imaging Analysis}

vGluT1-pHluorin: Individual synaptic puncta were selected randomly after $\mathrm{NH}_{4} \mathrm{Cl}$ perfusion and all quenched pHluroin probes were unmasked. Single vesicle events were analyzed as reported in Leitz et al. (2011) and Chanaday et al. (2018). Dwell times were calculated as the time between the initial fluorescence step and the start of fluorescence decay (using the first derivative as parameter). Single vesicle events were analyzed offline with Matlab. For $20 \mathrm{~Hz}$ stimulation, amplitude measurement and single exponential decay fitting (using Levenberg-Marquardt least sum of squares minimizations) were performed offline in Clampfit.

FM dye: Individual puncta were selected randomly after initial washout of excess dye not endocytosed. Synaptic pool size was estimated by taking the difference of the average fluorescence of individual puncta prior to $90 \mathrm{mM} \mathrm{K}^{+}$stimulation from the average fluorescence at the end of the second $90 \mathrm{mM} \mathrm{K}^{+}$stimulation. The rate of FM dye release was calculated by fitting the decay in fluorescence in response to the initial $90 \mathrm{mM} \mathrm{K}^{+}$tyrode stimulation to a single exponential decay function using Levenberg-Marquardt least sum of squares minimizations.

\section{Statistical Analysis}

Statistical analyses were performed with Graphpad Prism 6 software using one of the following tests: Student's ordinary t-test, Student's paired t-test, Two-way RM ANOVA and Kolmogorov-Smirnov test. Error bars represent SEM.

\section{Acknowledgements}

We would like to thank Drs. Helmut Kramer (UT Southwestern) and Sandy Schmid (UT

Southwestern) for sharing reagents as well as for their critical insight into this project. This work was supported by National Institute of Mental Health grants, MH081060 and MH070727 (LMM), 
bioRxiv preprint doi: https://doi.org/10.1101/2020.06.12.147975; this version posted June 12, 2020. The copyright holder for this preprint (which was not certified by peer review) is the author/funder, who has granted bioRxiv a license to display the preprint in perpetuity. It is made available under aCC-BY 4.0 International license.

502 and MH66198 (ETK).

503

504 Competing interests

505 The authors declare no competing interests.

506

507

508 


\section{References}

Antonny, B., Burd, C., De Camilli, P., Chen, E., Daumke, O., Faelber, K., Ford, M., Frolov, V.A., Frost, A., Hinshaw, J.E., et al. (2016). Membrane fission by dynamin: what we know and what we need to know. EMBO J 35, 2270-2284.

Armbruster, M., Messa, M., Ferguson, S.M., De Camilli, P., and Ryan, T.A. (2013). Dynamin phosphorylation controls optimization of endocytosis for brief action potential bursts. Elife 2, e00845.

Artalejo, C.R., Elhamdani, A., and Palfrey, H.C. (2002). Sustained stimulation shifts the mechanism of endocytosis from dynamin-1-dependent rapid endocytosis to clathrin- and dynamin-2-mediated slow endocytosis in chromaffin cells. Proc Natl Acad Sci U S A 99, 63586363.

Baldassarre, M., Pompeo, A., Beznoussenko, G., Castaldi, C., Cortellino, S., McNiven, M.A., Luini, A., and Buccione, R. (2003). Dynamin participates in focal extracellular matrix degradation by invasive cells. Mol Biol Cell 14, 1074-1084.

Cao, H., Chen, J., Awoniyi, M., Henley, J.R., and McNiven, M.A. (2007). Dynamin 2 mediates fluid-phase micropinocytosis in epithelial cells. Journal of cell science 120, 4167-4177.

Chanaday, N.L., and Kavalali, E.T. (2017). How do you recognize and reconstitute a synaptic vesicle after fusion? F1000Res 6, 1734.

Chanaday, N.L., and Kavalali, E.T. (2018). Optical detection of three modes of endocytosis at hippocampal synapses. eLife 7, pii: e36097.

Cousin, M.A., and Robinson, P.J. (2000). Ca(2+) influx inhibits dynamin and arrests synaptic vesicle endocytosis at the active zone. J Neurosci 20, 949-957.

Douthitt, H.L., Luo, F., McCann, S.D., and Meriney, S.D. (2011). Dynasore, an inhibitor of dynamin, increases the probability of transmitter release. Neuroscience 172, 187-195.

Evergren, E., Zotova, E., Brodin, L., and Shupliakov, O. (2006). Differential efficiency of the endocytic machinery in tonic and phasic synapses. Neuroscience 141, 123-131.

Fan, F., Funk, L., and Lou, X. (2016). Dynamin 1- and 3-Mediated Endocytosis Is Essential for the Development of a Large Central Synapse In Vivo. J Neurosci 36, 6097-6115.

Ferguson, S.M., Brasnjo, G., Hayashi, M., Wolfel, M., Collesi, C., Giovedi, S., Raimondi, A., Gong, L.W., Ariel, P., Paradise, S., et al. (2007). A selective activity-dependent requirement for dynamin 1 in synaptic vesicle endocytosis. Science 316, 570-574.

\section{Ferguson, S.M., and De Camilli, P. (2012). Dynamin, a membrane-remodelling GTPase. Nat} Rev Mol Cell Biol 13, 75-88.

Fornasiero, E.F., Mandad, S., Wildhagen, H., Alevra, M., Rammner, B., Keihani, S., Opazo, F., Urban, I., Ischebeck, T., Sakib, M.S., Fard, M.K., Kirli, K., Centeno, T.P., Vidal, R.O., Rahman, R.U., Benito, E., Fischer, A., Dennerlein, S., Rehling, P., Feussner, I., Bonn, S., Simons, M., Urlaub, H., and Rizzoli, S.O. (2018). Precisely measured protein lifetimes in the mouse brain reveal differences across tissues and subcellular fractions. Nat Commun. 9, 4230.

Gold, E.S., Underhill, D.M., Morrissette, N.S., Guo, J., McNiven, M.A., and Aderem, A. (1999). Dynamin 2 is required for phagocytosis in macrophages. J Exp Med 190, 1849-1856.

Grassart, A., Cheng, A.T., Hong, S.H., Zhang, F., Zenzer, N., Feng, Y., Briner, D.M., Davis, G.D., Malkov, D., and Drubin, D.G. (2014). Actin and dynamin2 dynamics and interplay during clathrin-mediated endocytosis. J Cell Biol 205, 721-735. 
552

553

554

555

556

557

558

559

560

561

562

563

564

565

566

567

568

569

570

571

572

573

574

575

576

577

578

579

580

581

582

583

584

585

586

587

588

589

590

591

592

593

594

Hua, Y., Woehler, A., Kahms, M., Haucke, V., Neher, E., and Klingauf, J. (2013). Blocking endocytosis enhances short-term synaptic depression under conditions of normal availability of vesicles. Neuron 80, 343-349.

Kavalali, E.T., and Jorgensen, E.M. (2014). Visualizing presynaptic function. Nat Neurosci 17, 10-16.

Kawasaki, F., Hazen, M., and Ordway, R. W. (2000). Fast synaptic fatigue in shibire mutants reveals a rapid requirement for dynamin in synaptic vesicle membrane trafficking. Nat. Neurosci. 3, 859-860.

Koenig, J.H., and Ikeda, K. (1989). Disappearance and reformation of synaptic vesicle membrane upon transmitter release observed under reversible blockage of membrane retrieval. J Neurosci 9, 3844-3860.

Lee, E., and De Camilli, P. (2002). Dynamin at actin tails. Proc Natl Acad Sci U S A 99, 161-166.

Leitz, J., and Kavalali, E.T. (2011). Ca(2)(+) influx slows single synaptic vesicle endocytosis. J Neurosci 31, 16318-16326.

Li, Y.C., Chanaday, N.L., Xu, W., and Kavalali, E.T. (2017). Synaptotagmin-1- and Synaptotagmin-7-Dependent Fusion Mechanisms Target Synaptic Vesicles to Kinetically Distinct Endocytic Pathways. Neuron 93, 616-631 e613.

Linares-Clemente, P., Rozas, J.L., Mircheski, J., Garcia-Junco-Clemente, P., Martinez-Lopez, J.A., Nieto-Gonzalez, J.L., Vazquez, M.E., Pintado, C.O., and Fernandez-Chacon, R. (2015). Different dynamin blockers interfere with distinct phases of synaptic endocytosis during stimulation in motoneurones. J Physiol 593, 2867-2888.

Liu, Y.W., Surka, M.C., Schroeter, T., Lukiyanchuk, V., and Schmid, S.L. (2008). Isoform and splice-variant specific functions of dynamin-2 revealed by analysis of conditional knock-out cells. Mol Biol Cell 19, 5347-5359.

Llobet, A., Gallop, J.L., Burden, J.J.E., Çamdere, G., Chandra, P., Vallis, Y,. Hopkins, C.R., Lagnado, L., and McMahon, H.T. (2011). Endophilin drives the fast mode of vesicle retrieval in a ribbon synapse. J Neurosci. 31, 8512-8519.

Lou, X., Fan, F., Messa, M., Raimondi, A., Wu, Y., Looger, L.L., Ferguson, S.M., and De Camilli, P. (2012). Reduced release probability prevents vesicle depletion and transmission failure at dynamin mutant synapses. Proc Natl Acad Sci U S A 109, E515-523.

McAdam, R.L., Varga, K.T., Jiang, Z., Young, F.B., Blandford, V., McPherson, P.S., Gong, L.W., and Sossin, W.S. (2015). The juxtamembrane region of synaptotagmin 1 interacts with dynamin 1 and regulates vesicle fission during compensatory endocytosis in endocrine cells. Journal of cell science 128, 2229-2235.

McCluskey, A., Daniel, J.A., Hadzic, G., Chau, N., Clayton, E.L., Mariana, A., Whiting, A., Gorgani, N.N., Lloyd, J., Quan, A., et al. (2013). Building a better dynasore: the dyngo compounds potently inhibit dynamin and endocytosis. Traffic 14, 1272-1289.

Min, L., Leung, Y.M., Tomas, A., Watson, R.T., Gaisano, H.Y., Halban, P.A., Pessin, J.E., and Hou, J.C. (2007). Dynamin is functionally coupled to insulin granule exocytosis. J Biol Chem 282, 33530-33536.

Orth, J.D., Krueger, E.W., Cao, H., and McNiven, M.A. (2002). The large GTPase dynamin regulates actin comet formation and movement in living cells. Proc Natl Acad Sci U S A 99, 167172. 
Park, R.J., Shen, H., Liu, L., Liu, X., Ferguson, S.M., and De Camilli, P. (2013). Dynamin triple knockout cells reveal off target effects of commonly used dynamin inhibitors. Journal of cell science 126, 5305-5312.

Raimondi, A., Ferguson, S.M., Lou, X., Armbruster, M., Paradise, S., Giovedi, S., Messa, M., Kono, N., Takasaki, J., Cappello, V., et al. (2011). Overlapping role of dynamin isoforms in synaptic vesicle endocytosis. Neuron 70, 1100-1114.

Renard, H.F., Simunovic, M., Lemière, J., Boucrot, E., Garcia-Castillo, M.D., Arumugam, S., Chambon, V., Lamaze, C., Wunder, C, Kenworthy, A.K., Schmidt, A.A., McMahon, H.T., Sykes, C., Bassereau, P., and Johannes, L. (2015). Endophilin-A2 functions in membrane scission in clathrin-independent endocytosis. Nature. 517, 493-496.

Rikhy, R., Kamat, S., Ramagiri, S., Sriram, V., and Krishnan, K.S. (2007). Mutations in dynaminrelated protein result in gross changes in mitochondrial morphology and affect synaptic vesicle recycling at the Drosophila neuromuscular junction. Genes Brain Behav 6, 42-53.

Saheki, Y., and De Camilli, P. (2012). Synaptic vesicle endocytosis. Cold Spring Harb Perspect Biol 4, a005645.

Schindelin J1, Arganda-Carreras I, Frise E, Kaynig V, Longair M, Pietzsch T, Preibisch S, Rueden C, Saalfeld S, Schmid B, Tinevez JY, White DJ, Hartenstein V, Eliceiri K, Tomancak P, Cardona A. (2012). Fiji: an open-source platform for biological-image analysis. Nat Methods. 2012 Jun 28;9(7):676-82.

Schlunck, G., Damke, H., Kiosses, W.B., Rusk, N., Symons, M.H., Waterman-Storer, C.M., Schmid, S.L., and Schwartz, M.A. (2004). Modulation of Rac localization and function by dynamin. Mol Biol Cell 15, 256-267.

Soykan, T., Maritzen, T., and Haucke, V. (2016). Modes and mechanisms of synaptic vesicle recycling. Curr Opin Neurobiol 39, 17-23.

Swadlow, H.A. (2003). Fast-spike interneurons and feedforward inhibition in awake sensory neocortex. Cereb Cortex 13, 25-32.

Tanifuji, S., Funakoshi-Tago, M., Ueda, F., Kasahara, T., and Mochida, S. (2013). Dynamin isoforms decode action potential firing for synaptic vesicle recycling. J Biol Chem 288, 1905019059.

Voglmaier, S.M., Kam, K., Yang, H., Fortin, D.L., Hua, Z., Nicoll, R.A., and Edwards, R.H. (2006). Distinct endocytic pathways control the rate and extent of synaptic vesicle protein recycling. Neuron 51, 71-84.

Watanabe, S., Rost, B.R., Camacho-Perez, M., Davis, M.W., Sohl-Kielczynski, B., Rosenmund, C., and Jorgensen, E.M. (2013). Ultrafast endocytosis at mouse hippocampal synapses. Nature 504, 242-247.

Wu, X.S., Lee, S.H., Sheng, J., Zhang, Z., Zhao, W.D., Wang, D., Jin, Y., Charnay, P., Ervasti, J.M., and Wu, L.G. (2016). Actin Is Crucial for All Kinetically Distinguishable Forms of Endocytosis at Synapses. Neuron 92, 1020-1035.

Xu, J., McNeil, B., Wu, W., Nees, D., Bai, L., and Wu, L.G. (2008). GTP-independent rapid and slow endocytosis at a central synapse. Nat Neurosci 11, 45-53. 


\section{Figure Legends}

\section{Figure 1}

A. Representative Western blot (top) and quantification (bottom) of dynamin 2 levels in control (left bands) and dynamin $2 \mathrm{KO}$ (right bands) neurons. There is $\sim 93 \%$ reduction in dynamin 2 protein levels at 18-20 DIV.

B. Example traces of normalized vGluT1-pHluorin responses to $20 \mathrm{~Hz} 100 \mathrm{AP}$ (5 s) stimulation in control (WT, black) and dynamin $2 \mathrm{KO}$ (red) hippocampal neuron cultures.

C. Cumulative distribution of the calculated fluorescence decay time constant $(\tau)$. Decay time courses are fit with a single exponential function for control (WT, black) and dynamin $2 \mathrm{KO}$ (red) neuronal cultures. WT: $\mathrm{N}=399$ boutons; dynamin $2 \mathrm{KO}: \mathrm{N}=340$ boutons. Inset: Average $\tau$ for WT (black) and dynamin $2 \mathrm{KO}$ (red) neurons ( $p=0.4081$ by Student's ordinary t-test).

D. Representative eEPSC traces from WT littermate controls (black) and dynamin $2 \mathrm{KO}$ (red) cultured hippocampal neurons.

E. Average eEPSC amplitudes from WT $(\mathrm{N}=10$, mean=1.5 $\mathrm{nA})$ and dynamin $2 \mathrm{KO}(\mathrm{N}=9$, mean $=1.3 \mathrm{nA}$ ) neurons are similar.

F. Sample eEPSC traces of the first 8 responses from WT (black) and dynamin $2 \mathrm{KO}$ (red) neurons to 400 stimuli applied at a $10 \mathrm{~Hz}$ frequency.

G. Normalized responses from WT (black, $\mathrm{N}=10$ ) and dynamin $2 \mathrm{KO}$ (red, $\mathrm{N}=9$ ) neurons after 400 pulses $10 \mathrm{~Hz}$ stimuli showing no effect of the absence of dynamin 2 on synaptic transmission.

H. Representative Western blot (top) and quantification (bottom) of total dynamin (using a pandynamin antibody) depicting the loss of all three dynamin isoforms (Cre treated cultures, orange) compared to littermate controls (empty L307 vector, blue). There is a substantial $(\sim 93 \%)$ reduction of dynamins 1, 2 and 3 after 15 DIV in cultured hippocampal neurons.

I. Sample electron micrograph images of Dnm $3 \mathrm{KO}$ and Dnm TKO synapses. White arrows: docked synaptic vesicles. Black bars $=200 \mu \mathrm{m}$.

J. Total number of synaptic vesicles (SV) per presynaptic area in Dnm $3 \mathrm{KO}$ (blue, $\mathrm{N}=41$ synapses, mean=65 SVs) and Dnm TKO synapses (orange, $\mathrm{N}=53$ synapses, mean=47 SVs; * $\mathrm{p}=0.0193$ by Student's ordinary t-test).

K. Number of docked synaptic vesicles in Dnm $3 \mathrm{KO}$ (blue, $\mathrm{N}=22$ synapses, mean=3 SVs) and Dnm TKO synapses (orange, $\mathrm{N}=19$ synapses, mean $=1.5 \mathrm{SVs}$; ${ }^{* *} \mathrm{p}=0.0039$ by Student's ordinary t-test). These results show a significant reduction in the sizes of the total pool and the readily releasable pool of synaptic vesicles after depletion of all dynamin isoforms.

L. Representative immunofluorescence images from Dnm $3 \mathrm{KO}$ and Dnm TKO neurites stained against vGAT (top panels) and vGluT1 (bottom panels). White bars $=2 \mu \mathrm{m}$.

M. Average number of inhibitory presynaptic boutons (VGAT positive) per $\mu \mathrm{m}^{2}$ in Dnm $3 \mathrm{KO}$ (blue, $\mathrm{N}=10$ images from 3 coverslips) and Dnm TKO cultures (orange, $\mathrm{N}=9$ images from 3 coverslips).

N. Average number of excitatory presynaptic boutons (vGluT1 positive) per $\mu \mathrm{m}^{2}$ in Dnm $3 \mathrm{KO}$ (blue, $\mathrm{N}=8$ images from 2 coverslips) and Dnm TKO cultures (orange, $\mathrm{N}=6$ images from 2 coverslips).

\section{Figure 2}


681

682

683

684

685

686

687

688

689

690

691

692

693

694

695

696

697

698

699

700

701

702

703

704

705

706

707

708

709

710

711

712

713

714

715

716

717

718

719

720

721

722

723

724

A. Sample vGluT1-pHluorin traces in Dnm $3 \mathrm{KO}$ (blue) and Dnm TKO (orange) boutons in response to $20 \mathrm{~Hz} 100 \mathrm{AP}(5 \mathrm{~s})$ stimulation $(\Delta \mathrm{F})$ and after perfusion of $20 \mathrm{mM} \mathrm{NH}_{4} \mathrm{Cl}\left(\mathrm{F}_{\mathrm{NH} 4 \mathrm{Cl}}\right)$. B. Percent distribution of the different retrieval types observed in Dnm TKO neurons following $20 \mathrm{~Hz}$ stimulation. $63 \%$ of Dnm TKO boutons decayed back to at least $70 \%$ of the initial increase in fluorescence $(\Delta \mathrm{F})$ indicating high (similar to control) retrieval of the fused synaptic vesicle proteins. $25 \%$ of Dnm TKO synapses had obstructed endocytosis since they decayed to less than $20 \%$ of the initial increase in fluorescence while the remaining $12 \%$ of Dnm TKO synapses showed partial variable efficiency in retrieval with decays between $20 \%-70 \%$ of the initial increase in fluorescence.

C. Cumulative histogram of vGluT1-pHluorin retrieval (\% of $\left.\Delta \mathrm{F}_{\text {ret }} / \Delta \mathrm{F}\right)$ after $20 \mathrm{~Hz} 100 \mathrm{AP}$ stimulation for Dnm $3 \mathrm{KO}$ (blue, $\mathrm{N}=1885$ boutons) and Dnm TKO (orange, N=967 boutons) showing an impairment in retrieval efficiency in the absence of all dynamin isoforms $(p<0.0001$, Kolmogorov-Smirnov test).

D. Cumulative distribution of response amplitudes ( $\Delta \mathrm{F}$ relative to $\mathrm{F}_{\mathrm{NH} 4 \mathrm{Cl}}$ ) after $20 \mathrm{~Hz} 100 \mathrm{AP}$ stimulation for Dnm $3 \mathrm{KO}$ (blue, $\mathrm{N}=1885$ boutons) and Dnm TKO (orange, $\mathrm{N}=967$ boutons) revealing a decrease of exocytosis in the absence of all dynamin isoforms $(p<0.0001$, Kolmogorov-Smirnov test).

E. Cumulative distribution of calculated decay time constant $(\tau)$ for Dnm $3 \mathrm{KO}$ (blue, mean=85 s) and Dnm TKO (orange, mean=137 s) showing a slowdown in endocytosis in neurons lacking dynamins 1,2 and 3 ( $p<0.0001$, Kolmogorov-Smirnov test).

F. Top: outline of FM dye release experiment. Synapses were preloaded with FM dye by incubating cultures in $45 \mathrm{mM} \mathrm{KCl}$ modified Tyrode's buffer containing $18 \mu \mathrm{M}$ FM5-95. Release of dye was induced by incubating cultures in $90 \mathrm{mM} \mathrm{KCl}$ modified Tyrode's buffer. Bottom: Average normalized $\Delta \mathrm{F}$ response in Dnm $3 \mathrm{KO}$ (blue, $\mathrm{N}=5$ coverslips) and Dnm TKO (orange, $\mathrm{N}=5$ coverslips) synapses after two successive stimulations with $90 \mathrm{mM} \mathrm{KCl}$ to induce release of FM5-95.

G. Cumulative distribution of the total change in fluorescence $(\Delta \mathrm{F})$ after 2 cycles of $90 \mathrm{mM} \mathrm{KCl}$ stimulation and FM5-95 dye release in Dnm 3 KO (blue) and Dnm TKO (orange) synapses showing a significant decrease in exocytosis in the absence of dynamins ( ${ }^{* * *} p<0.0001$, Kolmogorov-Smirnov test). Inset: average values of $\Delta \mathrm{F}$ after 2 cycles of $90 \mathrm{mM} \mathrm{KCl}$ stimulation. H. Cumulative histogram of the decay time constant $(\tau)$ from fitting FM5-95 fluorescence decay after the first $90 \mathrm{mM} \mathrm{KCl} \mathrm{stimulation,} \mathrm{in} \mathrm{Dnm} 3 \mathrm{KO}$ (blue) and Dnm TKO (orange) presynaptic boutons showing a slowdown in the kinetics of exocytosis in neurons lacking all dynamin isoforms ( ${ }^{* *} p<0.0001$ by Kolmogorov-Smirnov test). Inset: average $\tau$ values.

I. Analysis of single vesicle fusion events in Dnm $3 \mathrm{KO}$ synapses. Top: two representative single vesicle fusion events monitored with vGluT1-pHluorin (black lines show the dwell time and the subsequent exponential decay of fluorescence). Bottom: average of all single vesicle event traces fitted with a double exponential decay (black line) revealing the two components of endocytosis (ultrafast $\sim 360 \mathrm{~ms}$; fast $\sim 8.1 \mathrm{~s}$ ).

J. Analysis of single vesicle fusion events in Dnm TKO synapses. Top: two representative single vesicle fusion events monitored with vGluT1-pHluorin (black lines show the dwell time and the subsequent exponential decay of fluorescence). Bottom: average of all single vesicle event traces fitted with a double exponential decay (black line) revealing the two components of endocytosis (ultrafast $\sim 360 \mathrm{~ms}$; fast $\sim 6.7 \mathrm{~s}$ ). There is not a major impact of removal of all 
764

765

766

767

768

769

dynamin isoforms in the kinetics of single synaptic vesicle retrieval and reacidification during low frequency stimulation.

K. Cumulative histogram of amplitudes for single synaptic vesicle fusion events in Dnm $3 \mathrm{KO}$ (blue, $\mathrm{N}=1565$ boutons) and Dnm TKO (orange, $\mathrm{N}=961$ boutons) show a similar distribution between both groups. Inset: average amplitude $(\Delta \mathrm{F})$ for Dnm $3 \mathrm{KO}$ (blue, mean=82.2 a.u.) and Dnm TKO (orange, mean=83.0 a.u., $p=0.9137$ by Kolmogorov-Smirnov test).

L. Cumulative distribution of dwell times for Dnm $3 \mathrm{KO}$ (blue, $\mathrm{N}=1565$ boutons) and Dnm TKO (orange, $\mathrm{N}=961$ boutons). The lack of all three dynamin isoforms does not impact the kinetics of single synaptic vesicle endocytosis. Inset: frequency histogram of dwell time duration for each experimental group ( $p=0.06752$ by Kolmogorov-Smirnov test).

M. Classification of dwell times into three categories: Rapid decay ( $<1 \mathrm{~s}$ dwell), Short decay (1 $10 \mathrm{~s}$ dwell) and Long dwell (> $10 \mathrm{~s}$ dwell). The fraction of total measured dwell times into each category are shown for Dnm $3 \mathrm{KO}$ (blue) and Dnm TKO (orange), showing no differences in the proportion of fast or slow endocytic events between the groups. When only events from synapses with defects in endocytosis after high frequency stimulation (yellow traces in $B$, yellow bars in $\mathrm{M}$ ) were considered for the analysis, no significant defects in dwell times (single synaptic vesicle endocytosis) were observed (yellow).

\section{Figure 3}

A. Analysis of single vesicle fusion events in Dmn $3 \mathrm{KO}$ synapses at extracellular $8 \mathrm{mM} \mathrm{Ca}{ }^{2+}$ concentration. Top: two representative single vesicle fusion events (blue) monitored with vGluT1pHluorin (black lines show the dwell time and the subsequent exponential decay of fluorescence). Bottom: average of all single vesicle event traces fitted with a double exponential decay (blue line) revealing the two components of endocytosis (ultrafast $\sim 310 \mathrm{~ms}$; fast $\sim 19 \mathrm{~s} ; \mathrm{N}=888$ boutons). B. Analysis of single vesicle fusion events in Dmn TKO synapses at extracellular $8 \mathrm{mM} \mathrm{Ca}^{2+}$ concentration. Top: two representative single vesicle fusion events (orange) monitored with vGluT1pHluorin (black lines show the dwell time and the subsequent exponential decay of fluorescence). Bottom: average of all single vesicle event traces fitted with a double exponential decay (orange line) revealing the two components of endocytosis (ultrafast $\sim 260 \mathrm{~ms}$; fast $\sim 19 \mathrm{~s} ; \mathrm{N}=226$ boutons). There is not a major impact of removal of all dynamin isoforms in the kinetics of single synaptic vesicle retrieval and reacidification during low frequency stimulation. Moreover, in the absence of dynamins the $\mathrm{Ca}^{2+}$-dependent slowdown of endocytosis occurs normally.

C. Cumulative distribution of single vesicle event amplitudes from Dnm $3 \mathrm{KO}$ (blue, $\mathrm{N}=888$ boutons) and Dnm TKO (orange, $\mathrm{N}=226$ boutons) synapses in $8 \mathrm{mM} \mathrm{Ca}^{2+}$. Inset: Average amplitude for Dnm 3 KO (blue, mean=83.0 a.u.) and Dnm TKO (orange, mean=81.2 a.u., $p=0.8619$ by KolmogorovSmirnov test) are similar between the two experimental groups.

D. Cumulative distribution of single vesicle event dwell times from Dnm $3 \mathrm{KO}$ (blue, $\mathrm{N}=888$ boutons) and Dnm TKO (orange, $\mathrm{N}=226$ boutons) synapses in extracellular $8 \mathrm{mM} \mathrm{Ca}^{2+}$. There is no effect in the kinetics of single synaptic vesicle endocytosis after removal of all dynamin isoforms. Inset: Histograms of dwell time duration for both experimental groups $(p=0.1149$ by Kolmogorov-Smirnov test).

\section{Figure 4}

A. Left, sample eIPSC traces after a single stimulus from littermate control Dnm $3 \mathrm{KO}$ (blue) and Dnm TKO (orange) neurons. Right, average elPSC amplitudes of littermate control Dnm 3 KO 
812

813

(blue, mean=1.4 nA, N=31) and Dnm TKO (orange, mean =0.8 nA, N=27) hippocampal neuron cultures ( ${ }^{* *} p=0.0172$, Student's ordinary t-test). There is a decrease in amplitude in Dnm TKO neurons indicating a reduction in release probability.

B. Representative traces of the first 8 elPSC responses to a $1 \mathrm{~Hz} 30 \mathrm{AP}$ stimulus for Dnm $3 \mathrm{KO}$ (blue) and Dnm TKO (orange).

C. Normalized elPSC amplitudes after $1 \mathrm{~Hz} 30$ AP stimulus for Dnm $3 \mathrm{KO}$ (blue, N=9) and Dnm TKO (orange, $N=12$ ) showing no differences between the groups ( $p=0.9917$, Two-way RM ANOVA).

D. Sample traces of the first 13 elPSC responses to a $10 \mathrm{~Hz} 400$ AP stimulus for Dnm $3 \mathrm{KO}$ (blue) and Dnm TKO (orange) neurons.

E. Normalized elPSC amplitudes after $10 \mathrm{~Hz} 400$ AP stimulus for Dnm $3 \mathrm{KO}$ (blue, N=8) and Dnm TKO (orange, $N=12$ ) revealing similar depression time course for both groups $(p=0.7167$, Two-way RM ANOVA).

F. Top: sample traces of the first 10, middle 12 and last 12 eIPSC responses to a $30 \mathrm{~Hz} 1000 \mathrm{AP}$ stimulus for Dnm $3 \mathrm{KO}$ (blue) and Dnm TKO (orange). Bottom: normalized elPSC amplitudes after $30 \mathrm{~Hz} 1000 \mathrm{AP}$ stimulus for Dnm $3 \mathrm{KO}$ (blue, $\mathrm{N}=11$ ) and Dnm TKO (orange, $\mathrm{N}=7$ ). There is a facilitation of release at the beginning of the stimulation train in Dnm TKO indicative of reduced release probability in this group ( $p=0.0017$, Two-way RM ANOVA).

G. Representative mIPSC recordings from Dnm $3 \mathrm{KO}$ (blue) and Dnm TKO (orange) neurons. H. Average mIPSC frequency for Dnm $3 \mathrm{KO}$ (blue, $\mathrm{N}=10$, mean=1.8 Hz) and Dnm TKO (orange, $\mathrm{N}=11$, mean=1.4 Hz) hippocampal neuron cultures reveals no effect on the rate of inhibitory spontaneous neurotransmitter release after depletion of all dynamins $(p=0.6636$, Student's ordinary t-test).

I. Cumulative distribution of the mIPSC amplitudes for Dnm $3 \mathrm{KO}$ (orange) and Dnm TKO (blue) showing a small but significant reduction in amplitudes ( $p<0.0001$, Kolmogorov Smirnov test).

\section{Figure 5}

A. Sample eEPSC traces after a single stimulus from littermate control Dnm $3 \mathrm{KO}$ (blue) and Dnm TKO (orange) neurons. Inset: Average eEPSC amplitudes of littermate control Dnm $3 \mathrm{KO}$ (blue, mean=1.2 nA, N=10) and Dnm TKO (orange, mean=0.1 nA, N=10) neuronal cultures revealing a severe reduction in release probability at excitatory synapses ( ${ }^{*} p=0.0003$, Student's ordinary t-test).

B. Representative traces of the first 7 eEPSC responses to a $1 \mathrm{~Hz} 30$ AP stimulus for Dnm $3 \mathrm{KO}$ (blue) and Dnm TKO (orange). Arrows indicate application of stimulus, note the abnormal presence of failures in Dnm TKO trace.

C. Normalized eEPSC amplitudes after $1 \mathrm{~Hz} 30$ AP stimulus for Dnm $3 \mathrm{KO}$ (blue, N=10) and Dnm TKO (orange, $\mathrm{N}=10$ ) showing facilitation in $\mathrm{Dnm}$ TKO consistent with reduced release probability ( $p=0.0064$, Two-way RM ANOVA).
D. Sample traces of the first 13 eEPSC responses to a $10 \mathrm{~Hz} 400$ AP stimulus for Dnm $3 \mathrm{KO}$ (blue) and Dnm TKO (orange).

E. Normalized eEPSC amplitudes after $10 \mathrm{~Hz} 400$ AP stimulus for Dnm $3 \mathrm{KO}$ (blue, N=7) and Dnm TKO (orange, $\mathrm{N}=8$ ), depletion of all dynamin isoforms has a severe negative effect in the probability of release at excitatory synapses ( $p=0.0182$, Two-way RM ANOVA).
F. Representative mEPSC recordings from Dnm $3 \mathrm{KO}$ (blue) and Dnm TKO (orange) neurons. 
814 G. Average mEPSC frequency for Dnm $3 \mathrm{KO}$ (blue, $\mathrm{N}=10$, mean=1.74 Hz) and Dnm TKO

815 (orange, $\mathrm{N}=11$, mean $=0.76 \mathrm{~Hz}$ ) hippocampal neuron cultures revealing a $>2$-fold reduction in the

816 rate of spontaneous release $\left({ }^{*} p=0.0353\right.$, Student's ordinary t-test).

817 H. Cumulative distribution of mEPSC amplitudes for Dnm $3 \mathrm{KO}$ (blue) and Dnm TKO (orange)

818 showing no significant difference between the groups $(p<0.0001$, Kolmogorov-Smirnov test).

819

$820 \quad$ Figure 6.

821 A. Sample mEPSC traces from Dnm TKO hippocampal neuron cultures recorded before

822 (orange) and after (pink) treatment with Latrunculin A (20 $\mu \mathrm{M})$, CK-666 $(200 \mu \mathrm{M})$ and Mdivi-1 (50

$823 \mu \mathrm{M})$.

824 B. Average mEPSC frequency from Dnm TKO neurons before (orange) and after (pink)

825 treatment with Latrunculin $A(N=9, p=0.4856$, Student's paired t-test), CK-666 ( $N=6, p=0.7339$,

826 Student's paired t-test) and Mdivi-1 ( $N=8, p=0.3597$, Student's paired t-test). Actin dynamics,

827 Arp2/3 and the mitochondrial dynamin-related protein (DRP) do not mediate the remaining

828 excitatory neurotransmission after depletion of all dynamin isoforms. 
A
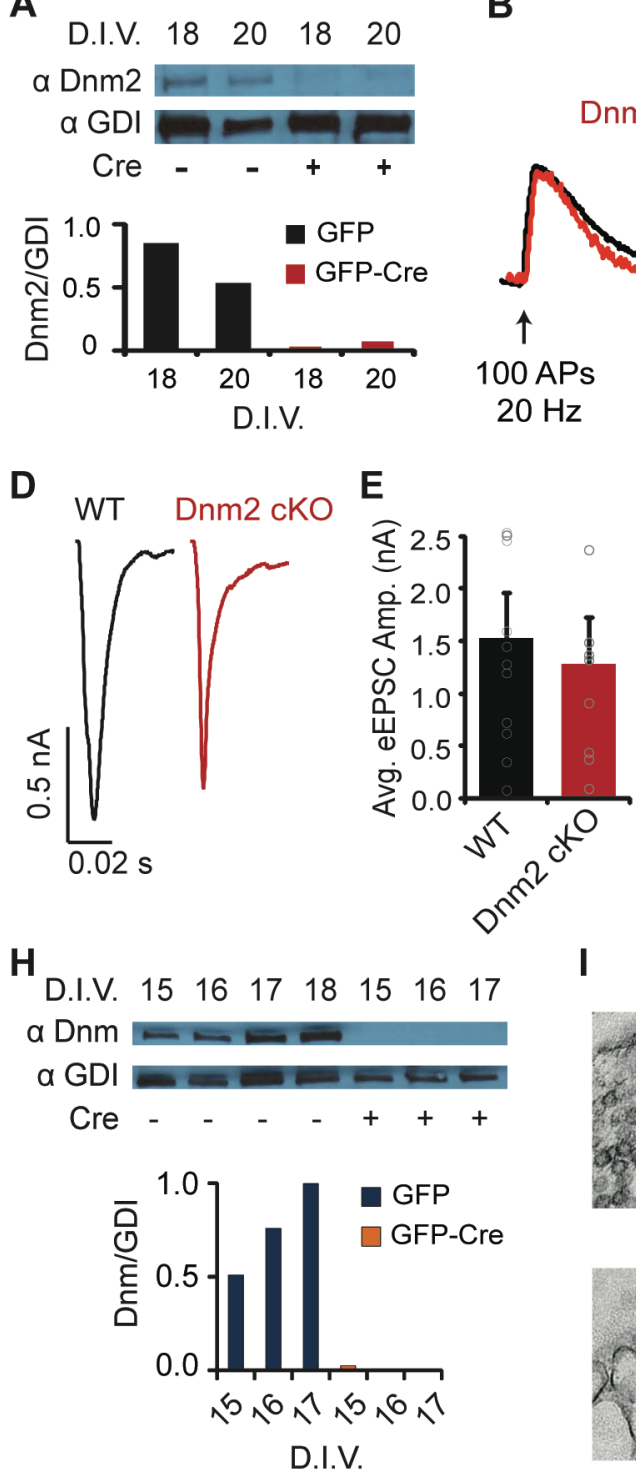

$\mathbf{L}$

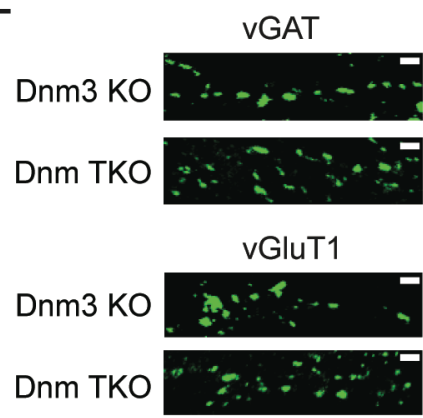

B

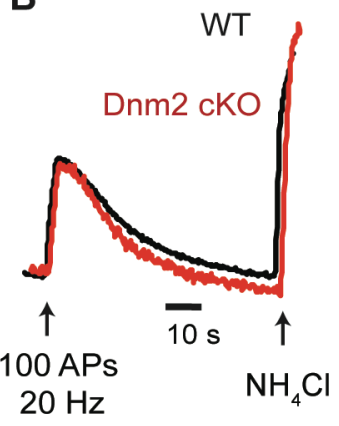

$\mathbf{F}$

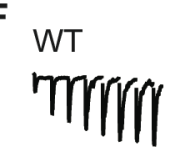

Dnm2 cKO

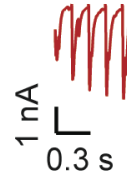

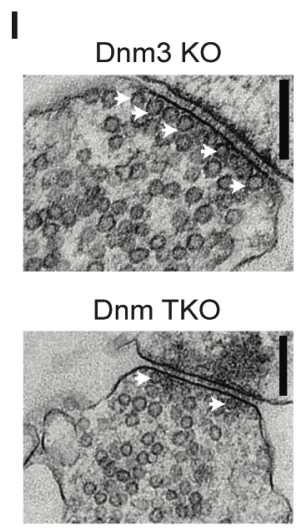

$$
J
$$

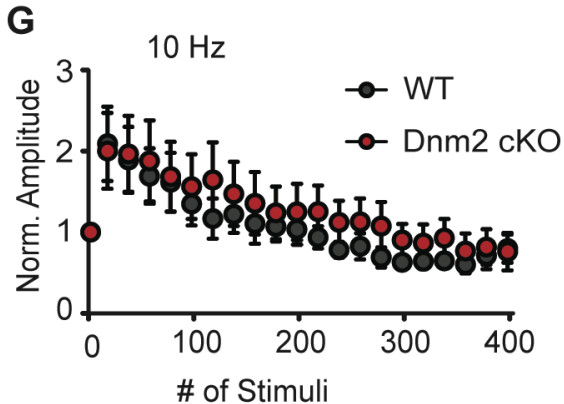

Total SV pool
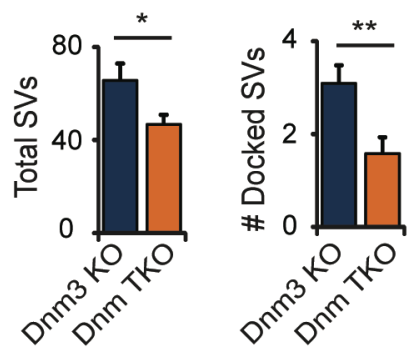

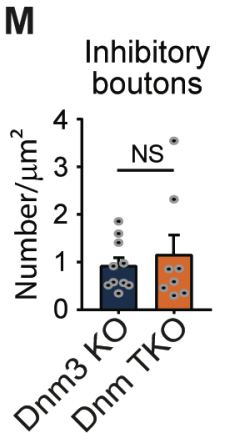

$\mathbf{N}$

Excitatory
boutons

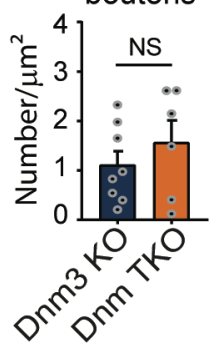


A
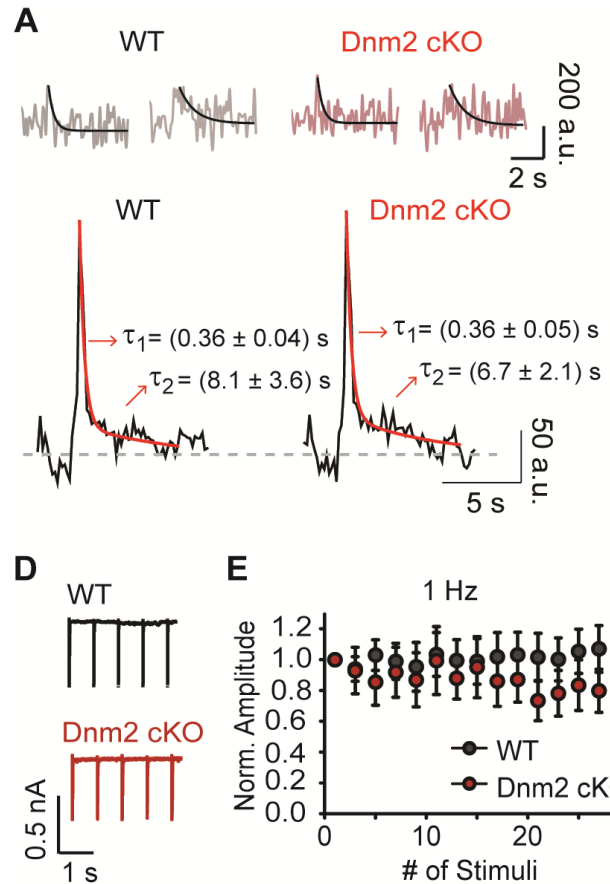

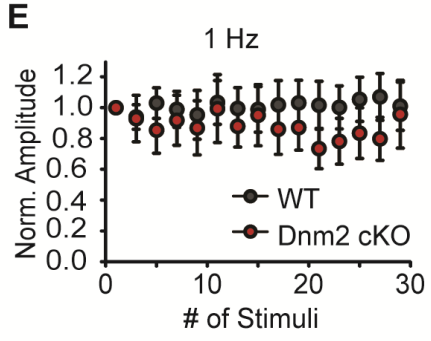

B

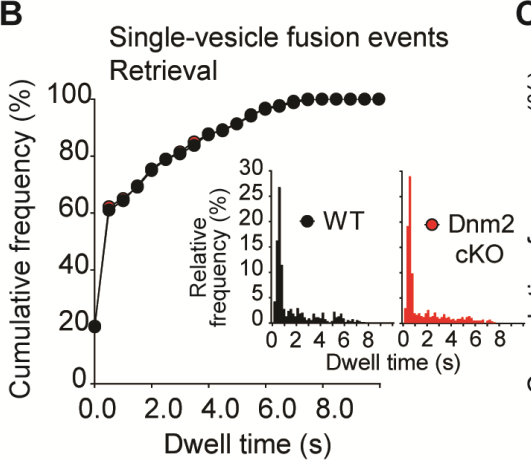

$\mathbf{F}$

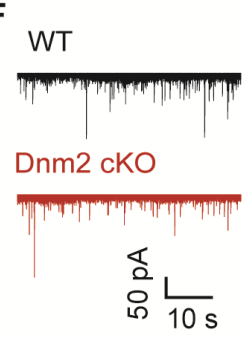

G
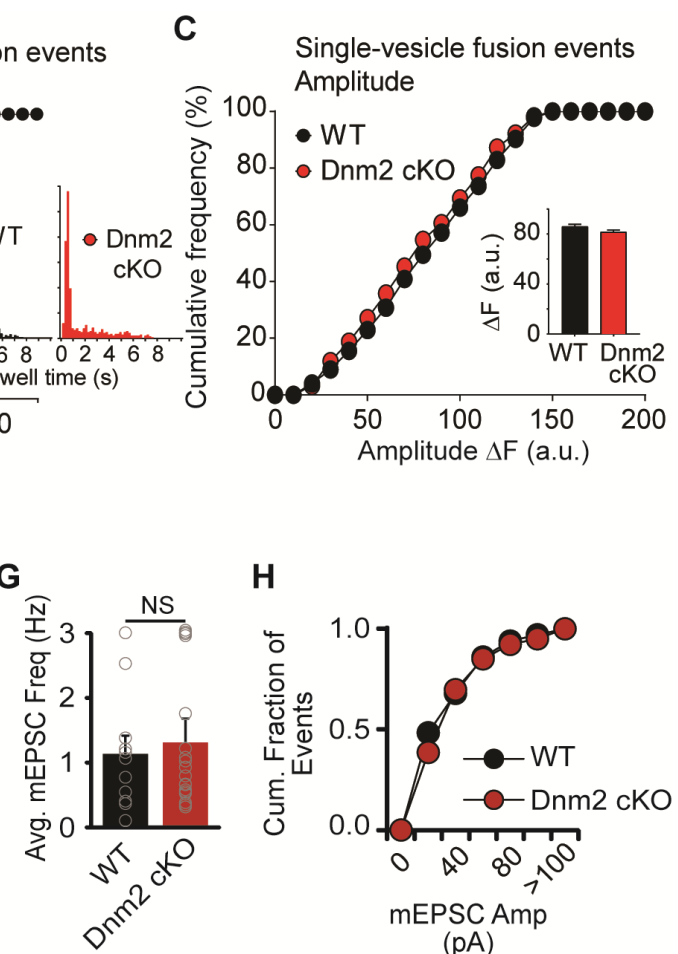

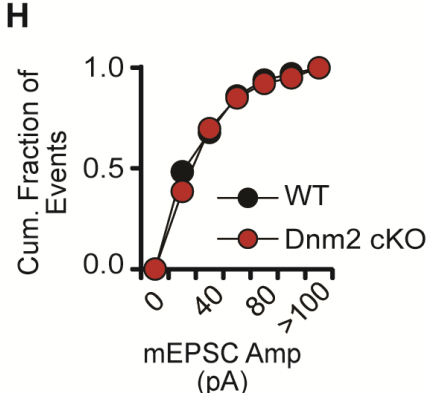

Figure 1 - figure supplement 1.

A. Analysis of single vesicle fusion events in Dnm $2 \mathrm{KO}$ synapses at extracellular $2 \mathrm{mM} \mathrm{Ca}^{2+}$ concentration. Top: two representative single vesicle fusion events monitored with vGluT1pHluorin for control (WT, grey) and Dnm 2 KO (pink; black lines show the exponential decay of fluorescence after fusion). Bottom: average of all single vesicle event traces for WT (left) and Dnm 2 KO (right) fitted with a double exponential decay (red line) revealing the two components of endocytosis (ultrafast $\sim 360 \mathrm{~ms}$; fast $\sim 6-8 \mathrm{~s}$ ). There is not a major impact of removal of Dnm 2 in the kinetics of single synaptic vesicle retrieval and reacidification during low frequency stimulation.

B. Cumulative distribution of single vesicle event dwell times from WT control (black, $N=429$ boutons) and Dnm $2 \mathrm{KO}$ (red, N=489 boutons) synapses. There is no effect in the kinetics of single synaptic vesicle endocytosis after removal of Dnm 2. Inset: Histograms of dwell time duration for both experimental groups ( $p=0.1391$ by Kolmogorov-Smirnov test).

c. Cumulative distribution of single vesicle event amplitudes from WT control (black, $N=429$ boutons) and Dnm $2 \mathrm{KO}$ (red, N=489 boutons) synapses. Inset: Average amplitude for WT (black, mean=86.1 a.u.) and Dnm $2 \mathrm{KO}$ (red, mean=81.7 a.u., p=0.8130 by KolmogorovSmirnov test) presynaptic terminals.

D. Sample traces of the first 5 responses from WT (black) and Dnm 2 KO (red) neurons to 30 stimuli applied at a $1 \mathrm{~Hz}$ frequency.

E. Normalized responses from WT (black, N=10) and Dnm $2 \mathrm{KO}$ (red, N=9) cultured hippocampal neurons after $1 \mathrm{~Hz} 30$ AP stimulation are similar for both groups ( $p=0.4385$, Twoway RM ANOVA).

F. Representative mEPSC traces from WT (black) and Dnm 2 KO (red) hippocampal neuron cultures. 
G. Average mEPSC frequency for WT (black, $\mathrm{N}=11$, mean=1.1 Hz) and Dnm $2 \mathrm{KO}$ (red, $\mathrm{N}=15$; mean=1.3 Hz) neurons showing no effect of the loss of dynamin 2 on presynaptic spontaneous release rate $(p=0.7500$, Student's ordinary t-test).

H. Cumulative distribution of mEPSC amplitude from WT (black) and Dnm $2 \mathrm{KO}$ (red) neuronal cultures. 

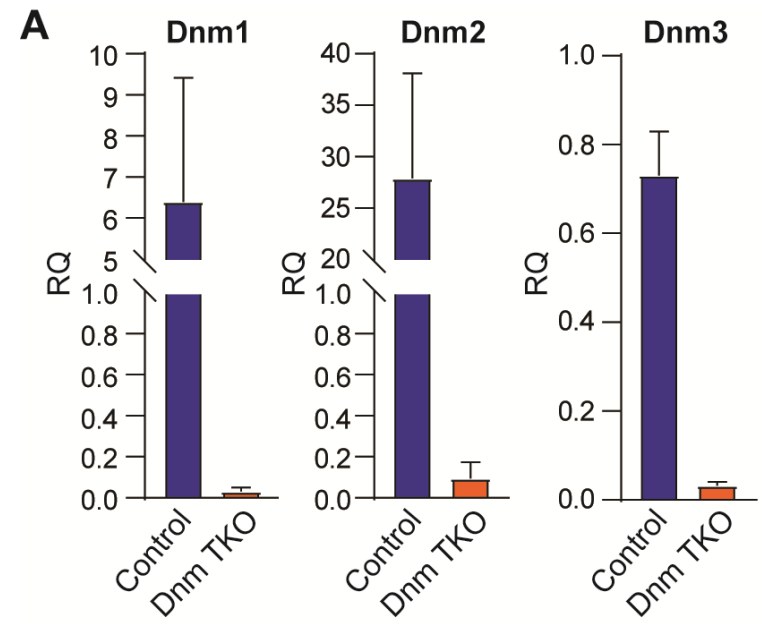

Figure 1 - figure supplement 2.

A. qRT-PCR of dynamins' mRNAs in cultured hippocampal neurons from Dnm TKO and littermate controls (controls were floxed Dnm1/2, Dnm3 heterozygous animals incubated with lentivirus expressing only GFP). Data comes from 2 independent cultures at DIV 19, each group was measured by triplicate. 
bioRxiv preprint doi: https://doi.org/10.1101/2020.06.12.147975; this version posted June 12, 2020. The copyright holder for this preprint (which was not certified by peer review) is the author/funder, who has granted bioRxiv a license to display the preprint in perpetuity. It is made
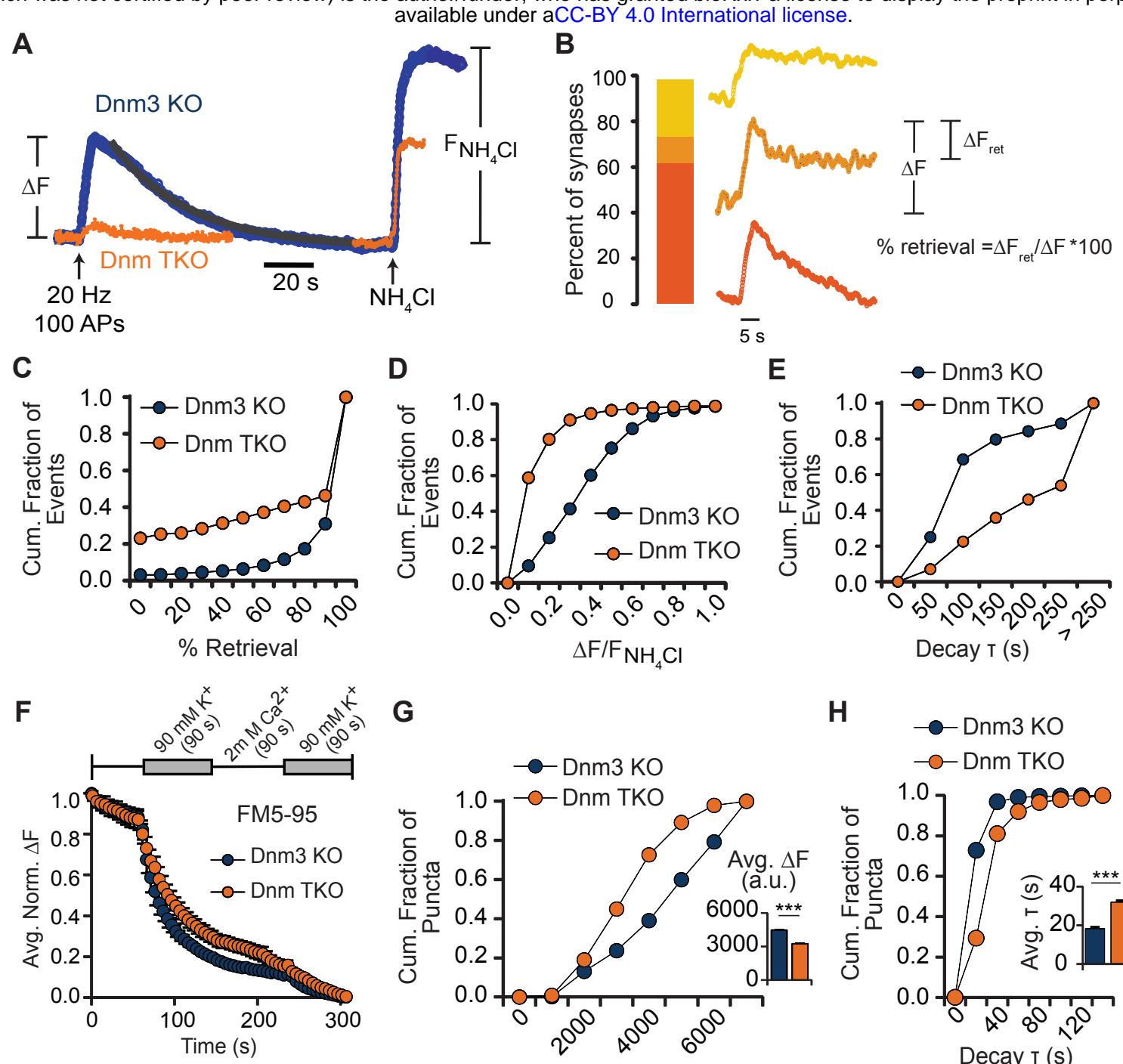

G

H
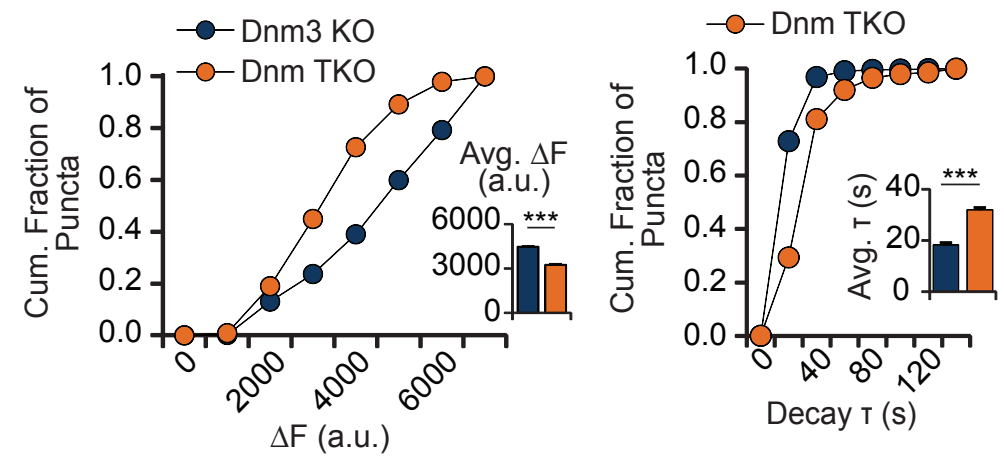

I

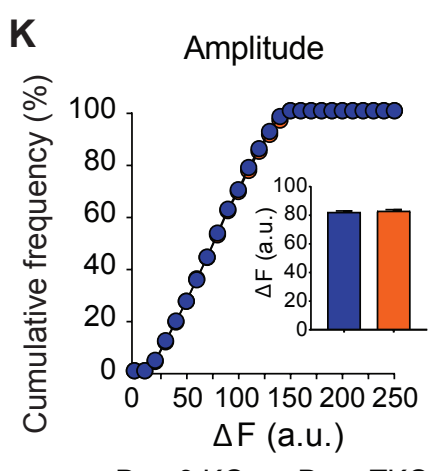

- Dnm3 KO O Dnm TKO

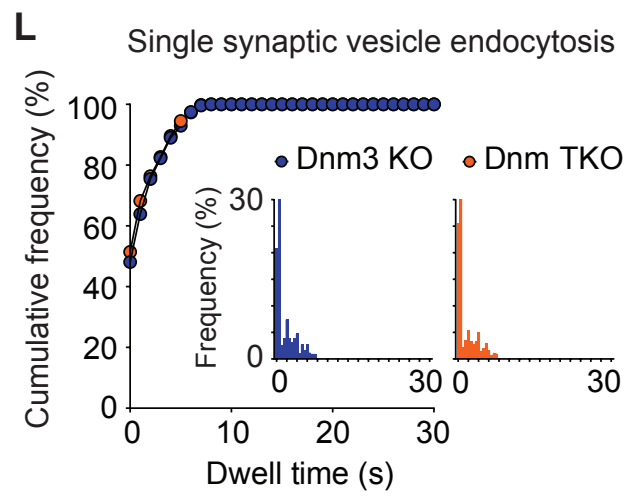

J Dnm TKO

M
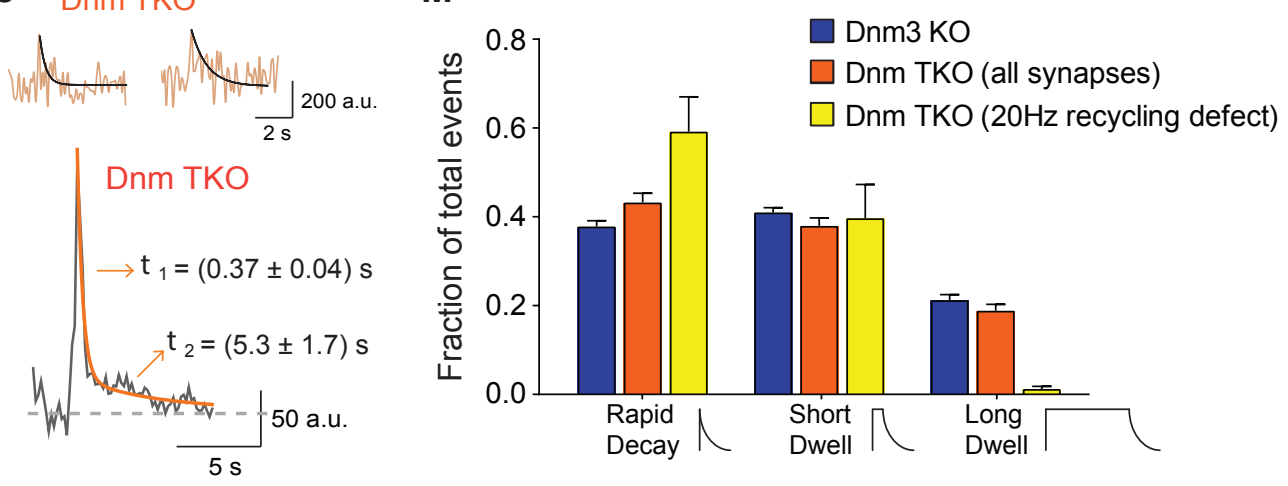
Single synaptic vesicle fusion events at $8 \mathrm{mM}$ extracellular $\mathrm{Ca}^{2+}$

A

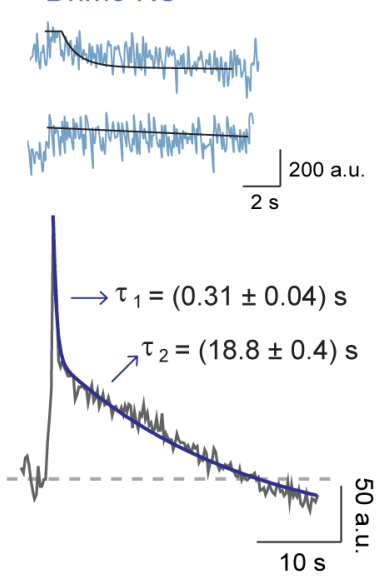

B

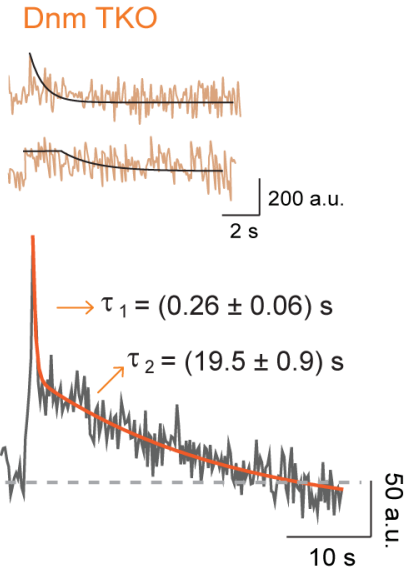

C

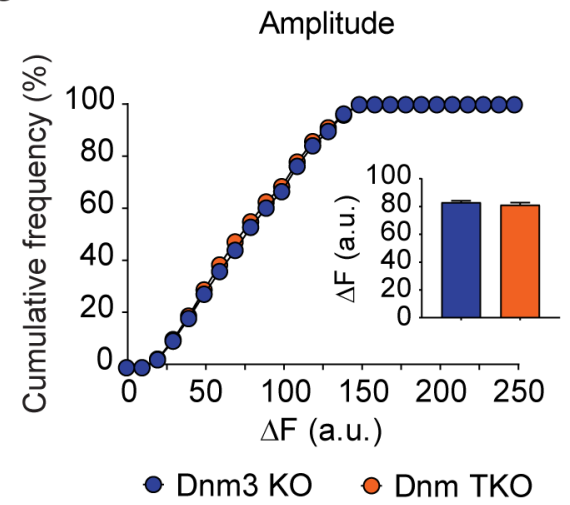

D

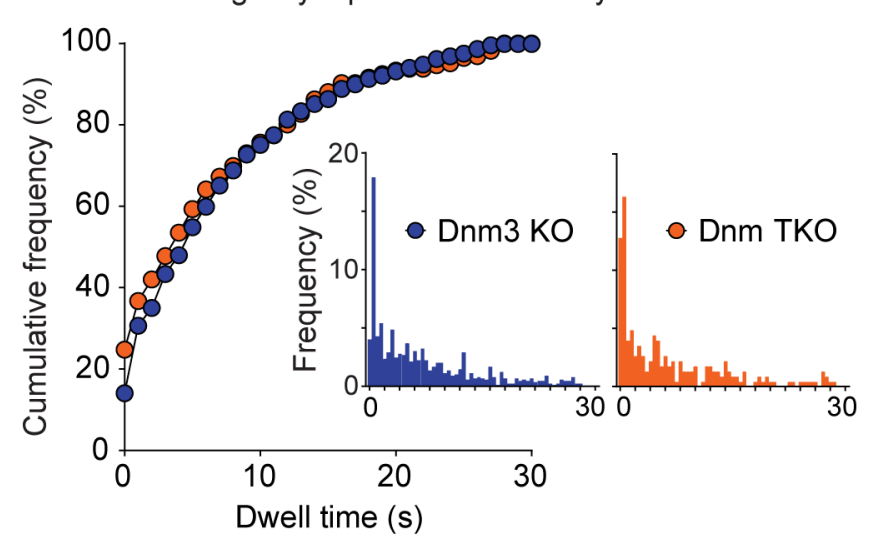



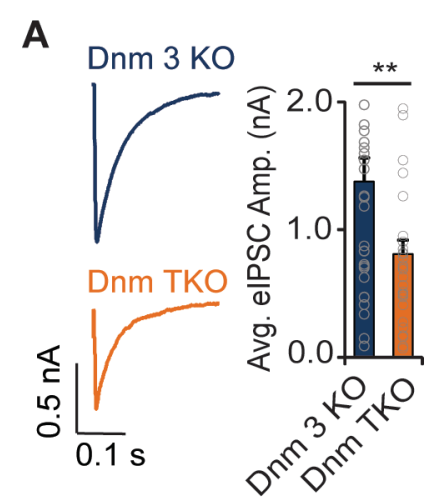

B

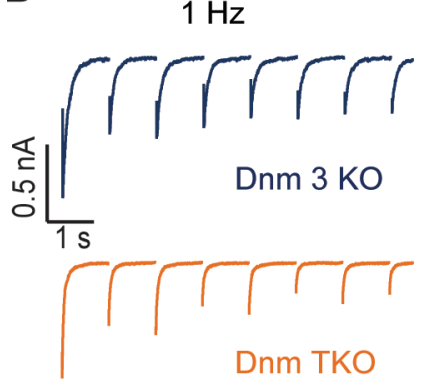

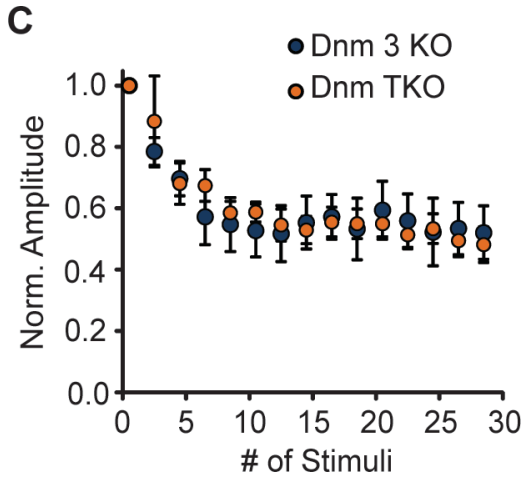

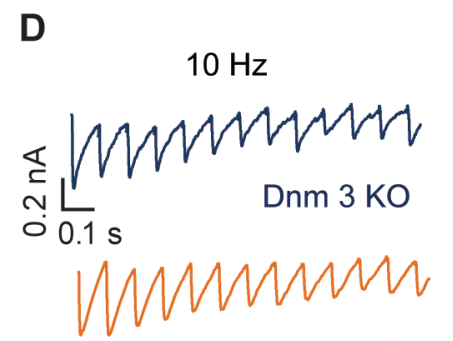

Dnm TKO
E

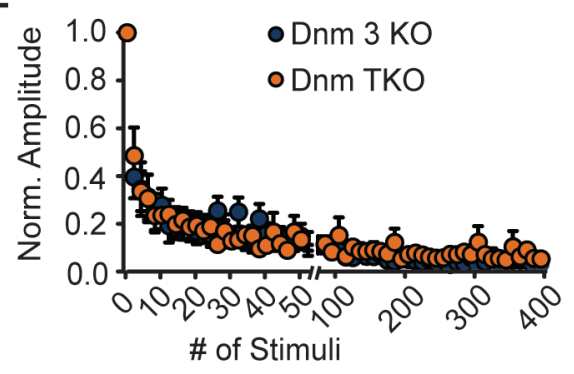

$\mathbf{F}$

$30 \mathrm{~Hz}$
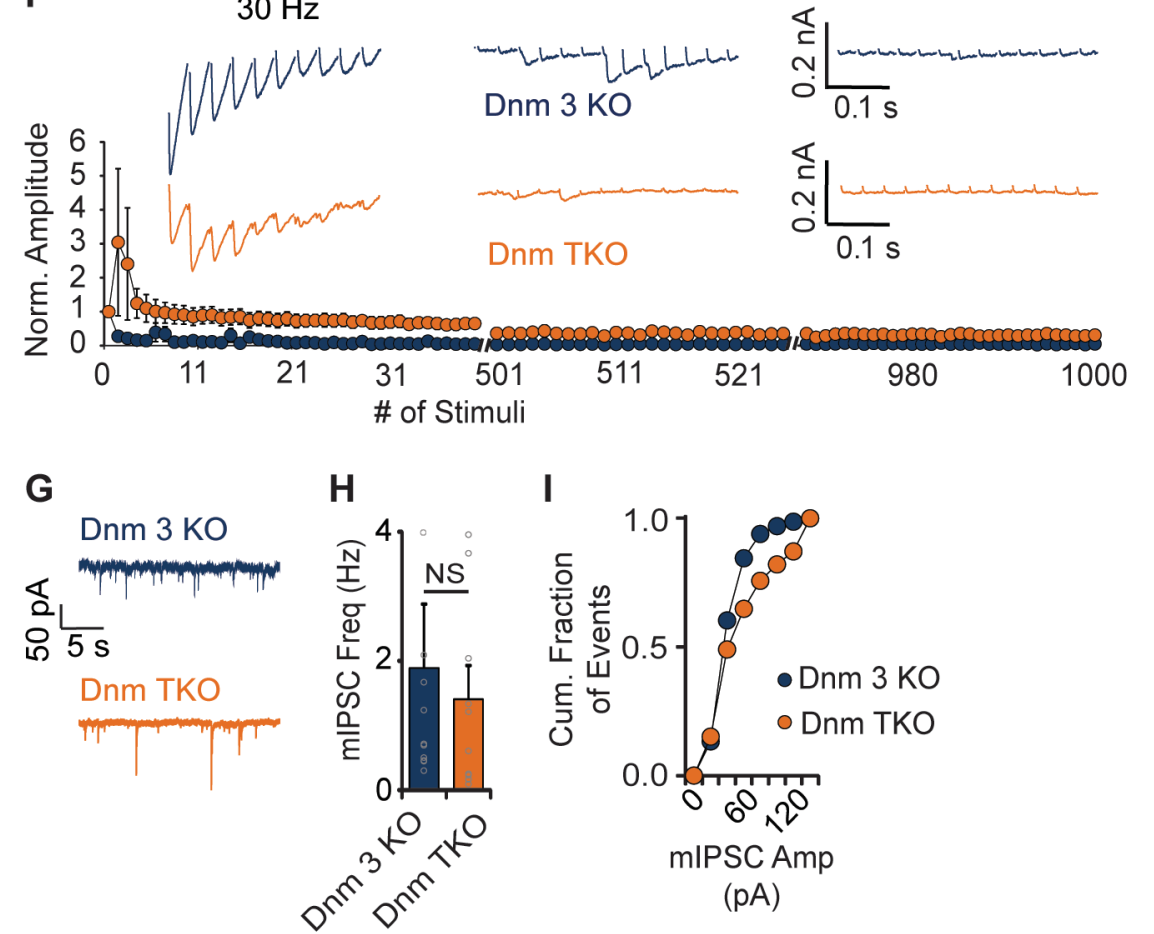
A

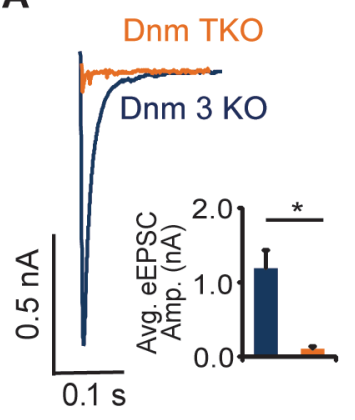

B $\quad 1 \mathrm{~Hz}$

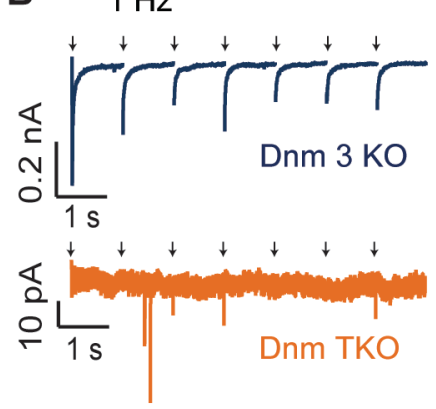

C

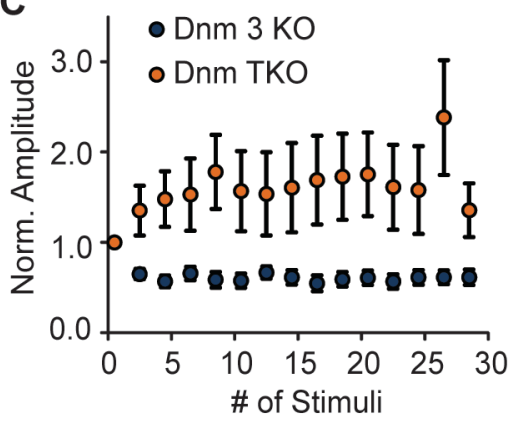

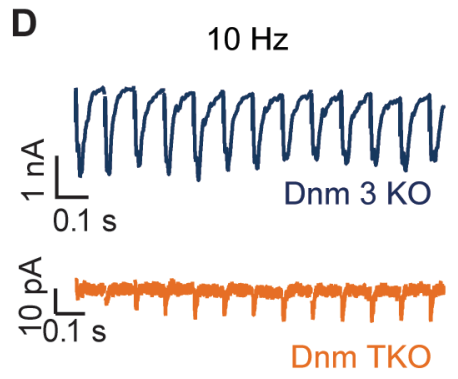

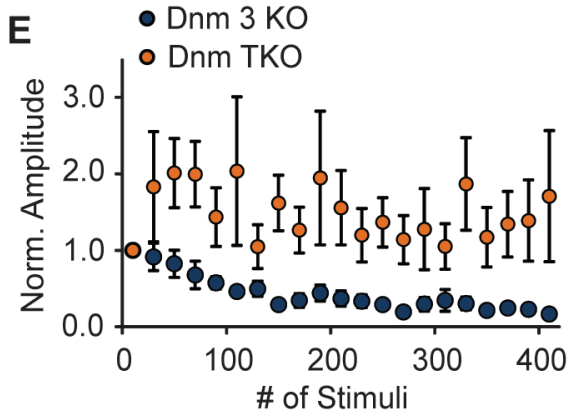

$\mathbf{F}$

Dnm $3 \mathrm{KO}$<smiles>C1CCCCC1</smiles>

Dnm TKO

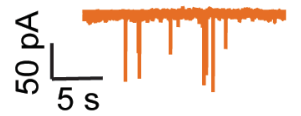

G

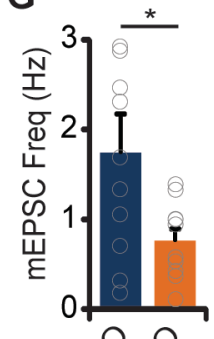

स्र

3 र
H

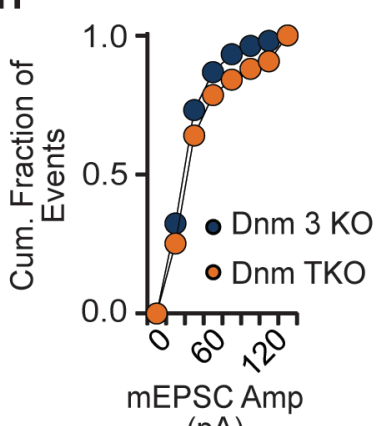

(pA) 
bioRxiv preprint doi: https://doi.org/10.1101/2020.06.12.147975; this version posted June 12, 2020. The copyright holder for this preprint (which was not certified by peer review) is the author/funder, who has granted bioRxiv a license to display the preprint in perpetuity. It is made available under aCC-BY 4.0 International license.

A

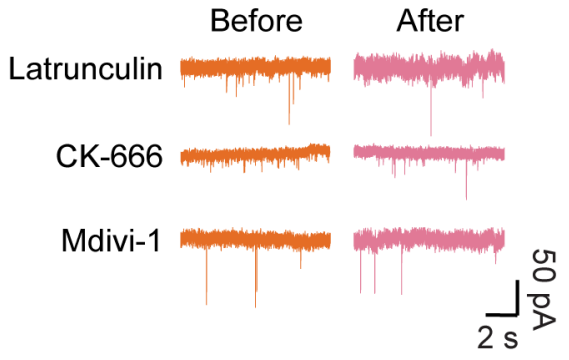

B

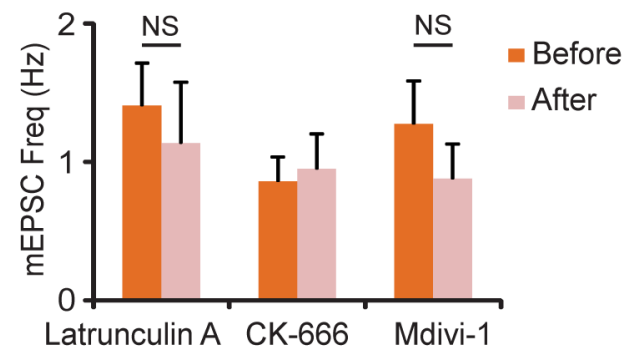

